\title{
Predicting intragranular misorientation distributions in polycrystalline metals using the viscoplastic self-consistent formulation
}

Zecevic, Miroslav ; Pantleon, Wolfgang; Lebensohn, Ricardo A.; McCabe, Rodney J.; Knezevic, Marko

Published in:

Acta Materialia

Link to article, DOI:

10.1016/j.actamat.2017.08.056

Publication date:

2017

Document Version

Peer reviewed version

Link back to DTU Orbit

Citation (APA):

Zecevic, M., Pantleon, W., Lebensohn, R. A., McCabe, R. J., \& Knezevic, M. (2017). Predicting intragranular misorientation distributions in polycrystalline metals using the viscoplastic self-consistent formulation. Acta Materialia, 140, 398-410. https://doi.org/10.1016/j.actamat.2017.08.056

\section{General rights}

Copyright and moral rights for the publications made accessible in the public portal are retained by the authors and/or other copyright owners and it is a condition of accessing publications that users recognise and abide by the legal requirements associated with these rights.

- Users may download and print one copy of any publication from the public portal for the purpose of private study or research.

- You may not further distribute the material or use it for any profit-making activity or commercial gain

- You may freely distribute the URL identifying the publication in the public portal 


\section{Accepted Manuscript}

Predicting intragranular misorientation distributions in polycrystalline metals using the viscoplastic self-consistent formulation

Miroslav Zecevic, Wolfgang Pantleon, Ricardo A. Lebensohn, Rodney J. McCabe,

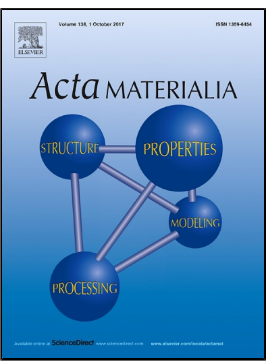
Marko Knezevic

PII: S1359-6454(17)30720-6

DOI: 10.1016/j.actamat.2017.08.056

Reference: AM 14017

To appear in: Acta Materialia

Received Date: 22 May 2017

Revised Date: 25 August 2017

Accepted Date: 26 August 2017

Please cite this article as: Miroslav Zecevic, Wolfgang Pantleon, Ricardo A. Lebensohn, Rodney J. McCabe, Marko Knezevic, Predicting intragranular misorientation distributions in polycrystalline metals using the viscoplastic self-consistent formulation, Acta Materialia (2017), doi: 10.1016/j. actamat.2017.08.056

This is a PDF file of an unedited manuscript that has been accepted for publication. As a service to our customers we are providing this early version of the manuscript. The manuscript will undergo copyediting, typesetting, and review of the resulting proof before it is published in its final form. Please note that during the production process errors may be discovered which could affect the content, and all legal disclaimers that apply to the journal pertain. 


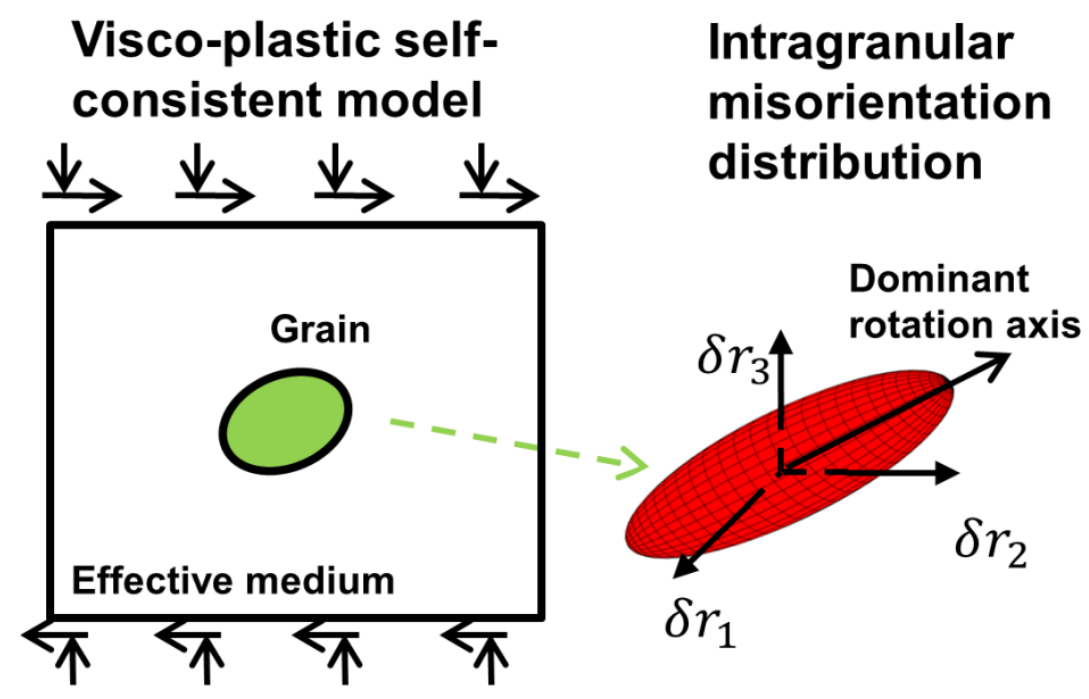

Dominant rotation axes direction

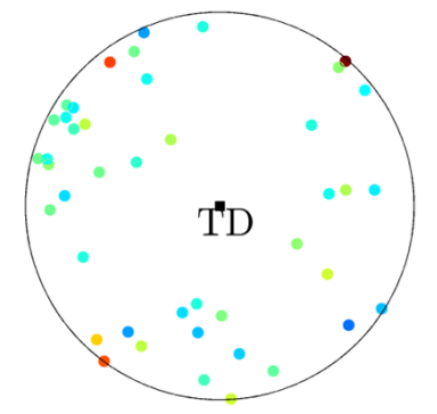

Experiment

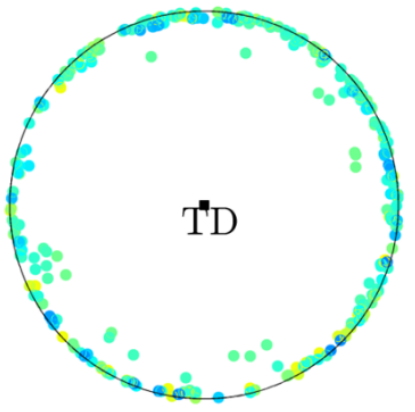

Simulation 


\title{
Predicting intragranular misorientation distributions in polycrystalline metals using the viscoplastic self-consistent formulation
}

\author{
Miroslav Zecevic ${ }^{1,2}$, Wolfgang Pantleon ${ }^{3}$, Ricardo A. Lebensohn², Rodney J. McCabe ${ }^{2}$, and \\ Marko Knezevic ${ }^{1, *}$ \\ ${ }^{1}$ Department of Mechanical Engineering, University of New Hampshire, Durham, NH 03824 \\ USA
}

${ }^{2}$ Materials Science and Technology Division, Los Alamos National Laboratory, Los Alamos, NM 87544

${ }^{3}$ Department of Mechanical Engineering, Technical University of Denmark, Produktionstorvet 425, 2800 Kgs. Lyngby, Denmark

\begin{abstract}
In a recent paper, we reported the methodology to calculate intragranular fluctuations in the instantaneous lattice rotation rates in polycrystalline materials within the mean-field viscoplastic self-consistent (VPSC) model. This paper is concerned with the time integration and subsequent use of these fluctuations to predict orientation-dependent misorientation distributions developing inside each grain representing the polycrystalline aggregate. To this end, we propose and assess two approaches to update the intragranular misorientation distribution within the VPSC framework. To illustrate both approaches, we calculate intragranular misorientations in facecentered cubic polycrystals deformed in tension and plane-strain compression. These predictions are tested by comparison with corresponding experiments for polycrystalline copper and aluminum, respectively, and with full-field calculations. It is observed that at sufficiently high strains some grains develop large misorientations that may lead to grain fragmentation and/or act as driving forces for recrystallization. The proposed VPSC-based prediction of intragranular misorientations enables modeling of grain fragmentation, as well as a more accurate modeling of texture using a computationally efficient mean-field approach, as opposed to computationally more expensive full-field approaches.
\end{abstract}

Keywords: Micromechanics; Crystal plasticity; Second order moments; Misorientation; Texture

\footnotetext{
${ }^{*}$ Corresponding author at: University of New Hampshire, Department of Mechanical Engineering, 33 Academic Way, Kingsbury Hall, W119, Durham, New Hampshire 03824, United States. Tel.: 603862 5179; fax: 603862 1865.

E-mail address: marko.knezevic@unh.edu (M. Knezevic).
} 


\section{Introduction}

During forming operations, polycrystalline metals are subjected to shape changes and plastic deformation that result in highly heterogeneous micromechanical fields (stress and strain fields) in the material [1]. It is well known that dislocation glide accommodates most of the imposed plastic deformation at the level of single grains. Crystallographic slip is potentially associated with local lattice rotations, which in turn induce anisotropy in the mechanical response by texture evolution and microstructure formation. Additionally, intra- and intergranular heterogeneities develop in the material, playing an important role in determining the deformation and hardening behavior, as well as the subsequent grain fragmentation and recrystallization of the deformed microstructure.

Intragranular orientation spreads have been related to the formation of transition bands, which are favorable places for recrystallization nucleation $[2,3]$. In addition, recrystallization nuclei forming near grain boundaries may have quite different crystallographic orientation compared to the average grain orientation due to large orientation variations developed across the grains [3, 4]. Therefore, accurate predictions of intragranular orientation distributions are crucial to model recrystallization processes. Furthermore, such misorientation predictions can improve the modelling of deformation textures and provide a physical basis for grain fragmentation.

Plastic deformation of polycrystalline metals is usually modeled using either full-field or meanfield approaches. Full-field models, for example, can be based on the crystal plasticity finite element (CPFE) method [5-9] or on the spectral fast Fourier transform (FFT)-based approach of Moulinec and Suquet [10], extended to polycrystals by Lebensohn [11]. These models are able to predict local micromechanical fields, including intragranular orientation gradients, resulting from grain-to-grain interactions, at the expense of a relatively high computational cost. Among meanfield models, self-consistent schemes, originally conceived for linear problems [12] and later extended to non-linear material's behavior [13-16], are widely used for simulating plastic deformation of polycrystalline materials considering grain interaction in an average sense. These non-linear homogenization-based models are computationally efficient, but most of them use information on first moments of the intragranular fields only, for the definition of a material with linearized behavior on which the self-consistency is actually imposed. In the last two decades, improved homogenization techniques have been developed that also take into account second moments of the micromechanical fields to define an optimized linearization $[17,18]$. However, until very recently this available information on second moments of the intragranular fields had not been used in calculations of intragranular misorientation spreads.

There have been numerous phenomenological attempts to predict the development of intragranular orientation spreads without the use of computationally expensive full-field approaches. Berveiller et al. [19] divided grains into homogeneous regions where plastic deformation occurred by single or multiple slip. They showed that these regions developed quite different orientations upon plastic deformation, indicating the formation of an orientation spread. 
Bolmaro et al. [20] represented each grain within the viscoplastic self-consistent (VPSC) model by two fragments to which random neighbors were assigned. Co-rotation of grain fragments and their respective neighbors was assumed, resulting in the development of local misorientation between fragments. This model was later used to initialize the local states to drive a recrystallization model [21]. The idea of co-rotation in VPSC was further extended by [22], representing each grain by six fragments, each one co-rotating with its own neighbor. In a similar approach developed by Toth et al. [23], a Taylor-type polycrystal model was used, in which each grain was divided into two regions, one at the center of the grain and another close to grain boundaries. In the latter, rotation was impeded by "friction" with the surrounding medium. Due to the difference in rotation between the two regions, lattice curvature was predicted and in turn used in a fragmentation model. Raabe [24] estimated the intragranular misorientation by assuming one part of the grain was next to a hard grain and thus deformed according to the Taylor model, while another part had a soft neighbor and consequently deformed according to the Sachs model. In [2] rotation rate fields were analyzed to determine the divergent and convergent orientations under different applied strains. A similar idea was developed by Raabe et al. [25] where the divergence of the rotation rate field was used to determine which orientations would be prone to develop strong orientation differences. Lee and Duggan [26] used an intragranular deformation banding model within the Taylor framework to simulate rolling textures of copper. A similar model was later used by Leffers [27] for simulating microstructure development in rolled aluminum. Butler and McDowell [28] introduced additional plastic rotations within the Taylor model to account for accumulation of geometrically necessary dislocations related to grain subdivision. In recent work Guo and Seefeldt [29] have used VPSC to model formation of reorientation bands and resulting grain fragmentation, caused by slip bands in neighboring grains. Slip rate within slip band for slip system $s$ was assumed to be proportional to mean slip rate value within grain and the coefficient of proportionality was assumed to be a fitting parameter. A majority of the described approaches have been introduced to improve texture predictions and to allow incorporation of the effect of grain fragmentation. Detailed analysis of the orientation spreads for a large number of grains and comparison with experimental measurements were generally missing in the aforementioned approaches.

In this work, the widely-used VPSC model [15] is extended to calculate intragranular misorientation spreads. The VPSC model is able to provide intragranular second moments of stress and strain rate fields $[30,31]$. In turn, this information can be used to calculate second moments of the rotation rates within individual grains [32]. In this paper, the latter information is further utilized to calculate corresponding intragranular misorientation spreads. In doing so, we formulate two algorithms to predict accumulation of intragranular misorientations based on time integration of first and second moments of the rotation rate fields. The two approaches are applied to uniaxial tension of copper and plane-strain compression of aluminum. The predictions are compared with experimental measurements and also with predictions using the full-field viscoplastic FFT-based (VPFFT) model. Considering the approximations involved in the 
formulation of the proposed mean-field model, reasonable agreement with experiments and the VPFFT model predictions is demonstrated for both approaches.

\section{Modeling framework}

In what follows we provide a short summary of the VPSC model, including the expressions to calculate second moments of the micromechanical fields in the grains. In our notation, tensors are denoted by bold letters while scalars and tensor components are indicated in italics and not bold. The contracted product and the tensor product between two tensors are denoted by ":" and “ $\bigotimes$ ", respectively. The constitutive relationship between the viscoplastic strain rate, $\dot{\boldsymbol{\varepsilon}}$, and the deviatoric stress, $\boldsymbol{\sigma}$, at material point $\mathbf{x}$ is given by the rate-sensitivity equation:

$$
\dot{\boldsymbol{\varepsilon}}(\mathbf{x})=\sum_{s} \dot{\gamma}^{s}(\mathbf{x}) \mathbf{m}^{s}(\mathbf{x})=\dot{\gamma}_{0} \sum_{s}\left(\frac{\boldsymbol{\sigma}(\mathbf{x}): \mathbf{m}^{s}(\mathbf{x})}{\tau_{c}^{s}(\mathbf{x})}\right){ }^{n} \operatorname{sign}\left(\boldsymbol{\sigma}(\mathbf{x}): \mathbf{m}^{s}(\mathbf{x})\right) \mathbf{m}^{s}(\mathbf{x})
$$

The constitutive parameters $\tau_{c}^{s}, \dot{\gamma}_{0}$, and $n$ are the critical resolved shear stress of slip system $s$, a reference shear rate, and the inverse of the rate sensitivity; $\dot{\gamma}^{s}$ is the shear rate and $\mathbf{m}^{s}$ is the symmetric part of the Schmid tensor, given by:

$$
\mathbf{m}^{S}(\mathbf{x})=\frac{1}{2}\left(\mathbf{b}^{S}(\mathbf{x}) \otimes \mathbf{n}^{S}(\mathbf{x})+\mathbf{n}^{S}(\mathbf{x}) \otimes \mathbf{b}^{S}(\mathbf{x})\right)
$$

with $\mathbf{n}^{S}$ and $\mathbf{b}^{S}$ denoting, respectively, the slip plane normal and the Burgers vector of slip system $s$.

The plastic spin, $\dot{\boldsymbol{\omega}}^{p}$, at material point $\mathbf{x}$ is given by:

$$
\dot{\boldsymbol{\omega}}^{p}(\mathbf{x})=\sum_{s} \dot{\gamma}^{s}(\mathbf{x}) \boldsymbol{\alpha}^{S}(\mathbf{x})
$$

where $\boldsymbol{\alpha}^{S}(\mathbf{x})=\frac{1}{2}\left(\mathbf{b}^{S}(\mathbf{x}) \otimes \mathbf{n}^{S}(\mathbf{x})-\mathbf{n}^{S}(\mathbf{x}) \otimes \mathbf{b}^{S}(\mathbf{x})\right)$ is the antisymmetric part of the Schmid tensor of slip system $s$. By performing linearization of the nonlinear constitutive relationships, we obtain the following expressions:

$$
\begin{aligned}
& \dot{\boldsymbol{\varepsilon}}(\mathbf{x})=\mathbf{M}^{(r)}: \boldsymbol{\sigma}(\mathbf{x})+\dot{\boldsymbol{\varepsilon}}^{0(r)} \\
& \dot{\gamma}^{s}(\mathbf{x})=\eta^{s(r)} \tau^{s}(\mathbf{x})+\dot{g}^{0 s(r)}
\end{aligned}
$$

where $\tau^{S}(\mathbf{x})=\mathbf{m}^{S}(\mathbf{x}): \boldsymbol{\sigma}(\mathbf{x})$ is the resolved shear stress on slip system $s . \mathbf{M}^{(r)}$ and $\dot{\boldsymbol{\varepsilon}}^{0(r)}$ are the linearized compliance and the back-extrapolated strain rate for grain $r$, respectively, while $\eta^{s(r)}$ and $\dot{g}^{0 s(r)}$ are the linearized compliance and the back-extrapolated shear rate of slip system $s$ in grain $r$, respectively. These moduli depend on the adopted linearization procedure. In what follows, we assume an affine linearization [33]. 
In order to obtain the macroscopic response of the linear polycrystal, we perform a selfconsistent homogenization, which implies a similar linear constitutive relation for the effective medium:

$$
\dot{\mathbf{E}}=\overline{\mathbf{M}}: \boldsymbol{\Sigma}+\dot{\mathbf{E}}^{0},
$$

$\dot{\mathbf{E}}$ and $\boldsymbol{\Sigma}$ are the macroscopic strain rate and stress and $\overline{\mathbf{M}}$ and $\dot{\mathbf{E}}^{0}$ are the macroscopic compliance and back-extrapolated strain rate, respectively. Within the self-consistent scheme every grain $r$ is treated as an ellipsoidal inhomogeneity embedded in the effective medium. This problem is solved using the equivalent inclusion approach [34] and the following interaction equation can be derived [15]:

$$
\tilde{\boldsymbol{\varepsilon}}^{(r)}=-\tilde{\mathbf{M}}: \tilde{\boldsymbol{\sigma}}^{(r)},
$$

where $\tilde{\boldsymbol{\varepsilon}}$ and $\tilde{\boldsymbol{\sigma}}$ are the deviations of strain rate and stress in grain $r$ from the macroscopic values. The interaction tensor $\widetilde{\mathbf{M}}=(\mathbf{I}-\mathbf{S})^{-1}: \mathbf{S}: \overline{\mathbf{M}}$ is a function of the classical (symmetric) Eshelby tensor, $\mathbf{S}$, and the unknown macroscopic compliance can be derived from the self-consistent equations:

$$
\begin{aligned}
& \overline{\mathbf{M}}=\left\langle\mathbf{M}^{(r)} \mathbf{B}^{(r)}\right\rangle, \\
& \dot{\mathbf{E}}^{0}=\left\langle\mathbf{M}^{(r)} \mathbf{b}^{(r)}+\dot{\boldsymbol{\varepsilon}}^{0(r)}\right\rangle,
\end{aligned}
$$

where $\mathbf{B}^{(r)}$ and $\mathbf{b}^{(r)}$ are the stress localization tensors, which are functions of the microscopic and macroscopic moduli. The self-consistent equations are, therefore, implicit in $\overline{\mathbf{M}}$ and $\dot{\mathbf{E}}^{0}$ and need to be solved iteratively using a fixed-point method based on Eqs. (8-9). The reorientation rate of the ellipsoid is given by:

$$
\tilde{\boldsymbol{\omega}}^{(r)}=\Pi: \mathbf{S}^{-1}: \tilde{\boldsymbol{\varepsilon}}^{(r)}
$$

where $\Pi$ is the antisymmetric Eshelby tensor. Assuming that the applied macroscopic rigid body rotation rate is equal to zero (as is the case for all of the performed simulations in this work) the total rate of lattice rotation at the material point can be written as:

$$
\dot{\boldsymbol{\omega}}^{(r)}(\mathbf{x})=\tilde{\boldsymbol{\omega}}^{(r)}-\dot{\boldsymbol{\omega}}^{p}(\mathbf{x})
$$

Once the self-consistent scheme converges, second moments of stress within each grain $r$ can be calculated as [30]:

$$
\langle\boldsymbol{\sigma} \otimes \boldsymbol{\sigma}\rangle^{(r)}=\frac{2 \partial \tilde{U}_{T}}{c^{(r)} \partial \mathbf{M}^{(r)}},
$$

where $c^{(r)}$ is the grain volume fraction and $\tilde{U}_{T}$ represents the effective potential given by: 


$$
\tilde{U}_{T}=\frac{1}{2} \overline{\mathbf{M}}::(\boldsymbol{\Sigma} \otimes \boldsymbol{\Sigma})+\dot{\mathbf{E}}^{0}: \boldsymbol{\Sigma}+\frac{1}{2} \bar{G} .
$$

The additional term $\bar{G}$ is the energy under zero applied stress. The second moment of the lattice rotation-rate within grain $r$ can be calculated as [32]:

$$
\langle\dot{\boldsymbol{\omega}} \otimes \dot{\boldsymbol{\omega}}\rangle^{(r)}=\left\langle\dot{\boldsymbol{\omega}}^{p} \otimes \dot{\boldsymbol{\omega}}^{p}\right\rangle^{(r)}+\tilde{\boldsymbol{\omega}}^{(r)} \otimes \tilde{\boldsymbol{\omega}}^{(r)}-\tilde{\boldsymbol{\omega}}^{(r)} \otimes\left\langle\dot{\boldsymbol{\omega}}^{p}\right\rangle^{(r)}-\left\langle\dot{\boldsymbol{\omega}}^{p}\right\rangle^{(r)} \otimes \tilde{\boldsymbol{\omega}}^{(r)} .
$$

Eq. (5) implies linearity between the plastic spin and stress. As a result, an expression for the second moment of plastic spin, $\left\langle\dot{\boldsymbol{\omega}}^{p} \otimes \dot{\boldsymbol{\omega}}^{p}\right\rangle^{(r)}$ can be obtained as a function of the first and second moments of stress [32]:

$$
\begin{aligned}
& \left\langle\dot{\boldsymbol{\omega}}^{p} \otimes \dot{\boldsymbol{\omega}}^{p}\right\rangle^{(r)}=\left\{\sum_{k, k^{\prime}}\left(\eta^{k(r)} \eta^{k^{\prime}(r)}\right)\left(\boldsymbol{\alpha}^{k(r)} \otimes \boldsymbol{\alpha}^{k^{\prime}(r)}\right) \otimes\left(\mathbf{m}^{k(r)} \otimes \mathbf{m}^{k^{\prime}(r)}\right)\right\}::\langle\boldsymbol{\sigma} \otimes \boldsymbol{\sigma}\rangle^{(r)} \\
& +\left\{\sum_{k, k^{\prime}}\left(\boldsymbol{\alpha}^{k(r)} \otimes \boldsymbol{\alpha}^{k^{\prime}(r)}\right) \otimes\left[\left(\eta^{k(r)} \dot{g}^{0 k^{\prime}(r)}\right) \mathbf{m}^{k(r)}+\left(\eta^{k^{\prime}(r)} \dot{g}^{0 k(r)}\right) \mathbf{m}^{k^{\prime}(r)}\right]\right\}:\langle\boldsymbol{\sigma}\rangle^{(r)} \\
& +\sum_{k, k^{\prime}}\left(\boldsymbol{\alpha}^{k(r)} \otimes \boldsymbol{\alpha}^{k^{\prime}(r)}\right)\left(\dot{g}^{0 k(r)} \dot{g}^{0 k^{\prime}(r)}\right) .
\end{aligned}
$$

Since $\left\langle\dot{\boldsymbol{\omega}}^{p}\right\rangle^{(r)}=\dot{\boldsymbol{\omega}}^{p(r)}$ is calculated based on the mean value of stress, the remaining terms in Eq. (14) can be readily evaluated. The covariance [35] of lattice rotation rate fluctuations is given by:

$$
\langle\delta \dot{\boldsymbol{\omega}} \otimes \delta \dot{\boldsymbol{\omega}}\rangle^{(r)}=\langle\dot{\boldsymbol{\omega}} \otimes \dot{\boldsymbol{\omega}}\rangle^{(r)}-\langle\dot{\boldsymbol{\omega}}\rangle^{(r)} \otimes\langle\dot{\boldsymbol{\omega}}\rangle^{(r)}=\left\langle\dot{\boldsymbol{\omega}}^{p} \otimes \dot{\boldsymbol{\omega}}^{p}\right\rangle^{(r)}-\left\langle\dot{\boldsymbol{\omega}}^{p}\right\rangle^{(r)} \otimes\left\langle\dot{\boldsymbol{\omega}}^{p}\right\rangle^{(r)},
$$

where $\delta \dot{\boldsymbol{\omega}}(\mathbf{x})=\dot{\boldsymbol{\omega}}^{(r)}(\mathbf{x})-\left\langle\dot{\boldsymbol{\omega}}^{(r)}\right\rangle$ represents the fluctuation of the lattice rotation rate. Consequently, the fluctuation of the rotation rate within a grain is determined by the fluctuation of plastic spin since the reorientation rate of the ellipsoid does not vary within the grain.

Finally, we turn our attention to the problem of incrementally updating the orientation field within grain $r$ using the available expressions for the moments of the rotation rate field. We assume that the orientation field within grain $r$ at the beginning of the current time increment, $t$, is known. In order to obtain the expression for the first and second moments of the orientation field at the end of the time increment, $t+\Delta t$, two approaches are considered: (1) a Taylor expansion approximation of misorientations and (2) a discrete approximation of misorientations. The two approaches will be compared with each other, with experiments and full-field simulation results.

\subsection{Taylor expansion approximation of misorientations}

In what follows, we use (unit) quaternion representation of crystal orientations and rotations. Quaternions are represented in matrix form [36], so that matrix algebra and notation can be used in the derivations. The matrix representation of quaternions is recalled in Appendix A. The orientation update at a material point $\mathbf{x}$ is given by: 


$$
\begin{aligned}
& \mathbf{q}^{t+\Delta t}(\mathbf{x})=\mathbf{q}_{\text {inc }}^{t}(\mathbf{x}) \mathbf{q}^{t}(\mathbf{x}), \\
& \left.q^{t+\Delta t}(\mathbf{x})=\left(q_{i n c}^{t}\right)_{i k}(\mathbf{x}) q_{k j}^{t}(\mathbf{x}) \text { (in index notation, with indices going from } 1 \text { to } 4\right),
\end{aligned}
$$

where $\mathbf{q}_{\text {inc }}^{t}(\mathbf{x})$ represents the active incremental rotation (expressed in sample frame) at time $t$ and $\mathbf{q}^{t}(\mathbf{x})$ and $\mathbf{q}^{t+\Delta t}(\mathbf{x})$ represent the active rotations (also expressed in sample frame) that rotate the sample frame into alignment with the crystal frame at times $t$ and $t+\Delta t$, respectively. Next, we express the crystal orientation at each material point $\mathbf{x}$ composing the mean rotation of grain $r$ to which the material point belongs and the local misorientation with respect to this average:

$$
\mathbf{q}^{t+\Delta t}(\mathbf{x})=\delta \mathbf{q}^{t+\Delta t}(\mathbf{x}) \overline{\mathbf{q}}^{t+\Delta t(r)}
$$

where the bar over a quaternion denotes the mean rotation of the grain and the $\delta$ in front of a quaternion denotes the misorientation with respect to that mean. By assuming analogous relations for $\mathbf{q}_{\text {inc }}^{t}(\mathbf{x})$ and $\mathbf{q}^{t}(\mathbf{x})$ we get:

$$
\delta \mathbf{q}^{t+\Delta t}(\mathbf{x}) \overline{\mathbf{q}}^{t+\Delta t(r)}=\left(\delta \mathbf{q}_{i n c}^{t}(\mathbf{x}) \overline{\mathbf{q}}_{\text {inc }}^{t(r)}\right)\left(\delta \mathbf{q}^{t}(\mathbf{x}) \overline{\mathbf{q}}^{t(r)}\right)
$$

Assuming that $\overline{\mathbf{q}}^{t+\Delta t(r)} \approx \overline{\mathbf{q}}_{\text {inc }}^{t(r)} \overline{\mathbf{q}}^{t(r)}$ and right multiplying both sides of Eq. (19) by $\overline{\mathbf{q}}^{t+\Delta t(r)-1}$ leads to:

$$
\delta \mathbf{q}^{t+\Delta t}(\mathbf{x})=\delta \mathbf{q}_{i n c}^{t}(\mathbf{x}) \delta \mathbf{q}^{t, r o t}(\mathbf{x})
$$

where $\delta \mathbf{q}^{t, r o t}(\mathbf{x})=\overline{\mathbf{q}}_{\text {inc }}^{t(r)} \delta \mathbf{q}^{t}(\mathbf{x}) \overline{\mathbf{q}}_{\text {inc }}^{t(r)-1}$ is the rotated misorientation at time $t$. The misorientation at time $t, \delta \mathbf{q}^{t}(\mathbf{x})$, represents a physical quantity: the rotation from the mean orientation to the orientation at material point $\mathbf{x}$. The operation $\overline{\mathbf{q}}_{\text {inc }}^{t(r)}(\ldots) \overline{\mathbf{q}}_{\text {inc }}^{t(r)-1}$ rotates the quantity in parenthesis by $\overline{\mathbf{q}}_{\text {inc }}^{t(r)}$. Hence, the rotated misorientation, $\delta \mathbf{q}^{t, r o t}(\mathbf{x})$, represents the misorientation at time $t, \delta \mathbf{q}^{t}$ $(\mathbf{x})$, rotated in space by the mean increment in rotation, $\overline{\mathbf{q}}_{\text {inc }}^{t(r)}$. The misorientation angle with respect to the mean orientation of the grain associated to the quaternion $\delta \mathbf{q}^{t}(\mathbf{x})$ will not change during this rotation to $\delta \mathbf{q}^{t, r o t}(\mathbf{x})$. However, the misorientation axis associated to the rotated quaternion $\delta \mathbf{q}^{\text {trot }}(\mathbf{x})$ is physically rotated in space by the mean increment in rotation when compared to misorientation axis of $\delta \mathbf{q}^{t}(\mathbf{x})$.

To simplify the notation, in what follows we shall not explicitly write the spatial dependence of the quaternions with the material point $\mathbf{x}$. According to Eq. (20), $\delta \mathbf{q}^{t+\Delta t}$ is a function of two independent variables, $\delta \mathbf{q}_{\text {inc }}{ }^{t}$ and $\delta \mathbf{q}^{t, r o t}$, whose distributions, defined by their first and second moments, can be calculated. Explicit expressions for first and second moments of a function in terms of first and second moments of the corresponding variables are available only for linear functions of those variables. Therefore, we perform a first-order Taylor expansion of $\delta \mathbf{q}^{t+\Delta t}$ 
with respect to the variables $\delta \mathbf{q}_{\text {inc }}^{t}$ and $\delta \mathbf{q}^{t, r o t}$ so that we can derive explicit expressions for first and second moments of $\delta \mathbf{q}^{t+\Delta t}$. The natural choice of the reference points for this Taylor expansion are the mean values of variables $\delta \mathbf{q}_{i n c}{ }^{t}$ and $\delta \mathbf{q}^{t, r o t}$, which leads to the following linearized expression [37]:

$$
\begin{aligned}
& \delta \mathbf{q}^{t+\Delta t} \approx\left\langle\delta \mathbf{q}_{i n c}^{t}\right\rangle^{t(r)}\left\langle\delta \mathbf{q}^{t, r o t}\right\rangle^{(r)}+\left.\frac{\partial \delta \mathbf{q}^{t+\Delta t}}{\partial \delta \mathbf{q}_{i n c}^{t}}\right|_{\left\langle\left.\delta \mathbf{q}_{i n c}{ }^{t}\right|^{(r)},\left\langle\delta \mathbf{q}^{t, r o t}\right\rangle^{(r)}:\right.}:\left(\delta \mathbf{q}_{i n c}^{t}-\left\langle\delta \mathbf{q}_{i n c}{ }^{t}\right\rangle^{(r)}\right) \\
& +\left.\frac{\partial \delta \mathbf{q}^{t+\Delta t}}{\partial \delta \mathbf{q}^{t, r o t}}\right|_{\left\langle\delta \mathbf{q}_{i n c}{ }^{t}\right\rangle^{(r)},\left\langle\delta \mathbf{q}^{t, r o t}\right\rangle^{(r)}:\left(\delta \mathbf{q}^{t, r o t}-\left\langle\delta \mathbf{q}^{t, r o t}\right\rangle^{(r)}\right) .}
\end{aligned}
$$

The volume average of misorientations defined with respect to the mean orientation amounts to the identity rotation [38]: $\left\langle\left.\delta \mathbf{q}_{\text {inc }}{ }^{t}\right|^{(r)}=\left\langle\delta \mathbf{q}^{t, r o t}\right\rangle^{(r)}=\mathbf{I}\right.$, where $\mathbf{I}$ represents the identity matrix (Kronecker delta) in 4-D. The derivatives evaluated around the mean misorientation lead to (in index notation, with indices from 1 to 4 ):

$$
\begin{aligned}
& \frac{\partial \delta q_{i j}^{t+\Delta t}}{\partial\left(\delta q_{i n c}^{t}\right)_{k l} \mid} \mid\left\langle\left. q_{i n c}^{t}\right|^{(r)},\left\langle\delta q^{t, r o t}\right\rangle^{(r)}=I_{i k} I_{m l} \mid \delta q_{m j}^{t, r o t}\right\rangle=I_{i k} I_{j l}, \\
& \left.\left.\frac{\partial \delta q_{i j}^{t+\Delta t}}{\partial \delta q_{k l}^{t, r o t}}\right|_{\mid \delta q_{i n c}} ^{t}\right|^{(r)},\left\langle\delta q^{t, r o t}\right\rangle^{(r)}=\left\langle\left(\delta q_{i n c}^{t}\right)_{i m}\right\rangle I_{m k} I_{j l}=I_{i k} I_{j l} \cdot
\end{aligned}
$$

Substitution into Eq. (21) leads to:

$$
\delta \mathbf{q}^{t+\Delta t} \approx \delta \mathbf{q}_{i n c}^{t}+\delta \mathbf{q}^{t, r o t}-\mathbf{I} .
$$

Therefore, the linearized expression based on first-order Taylor expansion of Eq. (20) is a simple summation of unit quaternions. Starting with Eq. (20) and assuming that the misorientations with respect to the mean are infinitesimal rotations, the same expression (Eq. (24)) can be derived.

Since only three independent parameters are required to fully describe an arbitrary rotation and the vector parts of misorientation quaternions can be used for quantification of orientation spreads [38], we re-write the above equation (replacing " $\approx$ " by " $="$ ) in terms of the vector parts of the misorientation quaternions (see Appendix A):

$$
\delta \mathbf{r}^{t+\Delta t}=\delta \mathbf{r}_{\text {inc }}^{t}+\delta \mathbf{r}^{t, r o t} .
$$

The identity quaternion present in Eq. (24) vanishes since the vector part of identity quaternion is zero. Finally, the expressions for the first and second moments of the vector parts of misorientation quaternion are given as:

$$
\left\langle\delta \mathbf{r}^{t+\Delta t}\right\rangle^{(r)}=\left\langle\delta \mathbf{r}_{i n c}^{t}\right\rangle^{(r)}+\left\langle\delta \mathbf{r}^{t, r o t}\right\rangle^{(r)}=\mathbf{0}
$$




$$
\begin{aligned}
& \left\langle\delta \mathbf{r}^{t+\Delta t} \otimes \delta \mathbf{r}^{t+\Delta t}\right\rangle^{(r)}=\left\langle\delta \mathbf{r}_{i n c}^{t} \otimes \delta \mathbf{r}_{i n c}^{t}\right\rangle^{(r)}+\left\langle\delta \mathbf{r}^{t, r o t} \otimes \delta \mathbf{r}_{i n c}^{t}\right\rangle^{(r)}+\left\langle\delta \mathbf{r}_{i n c}^{t} \otimes \delta \mathbf{r}^{t, r o t}\right\rangle^{(r)}+ \\
& +\left\langle\delta \mathbf{r}^{t, r o t} \otimes \delta \mathbf{r}^{t, r o t}\right\rangle^{(r)} .
\end{aligned}
$$

The first moments of the vector parts of misorientation quaternions vanish because of our choice for the mean orientation value, which implies that the second moment is already centered. Consequently, the second moment of the vector parts of misorientation quaternions is a suitable quantity for characterization of the orientation spread [38].

As it was noted before, the quaternion $\delta \mathbf{q}^{t, r o t}$ is obtained by rotation of the quaternion $\delta \mathbf{q}^{t}$ in space by the mean increment in rotation $\overline{\mathbf{q}}_{\text {inc }}^{t(r)}$. Since we are now dealing with 3-dimensional Cartesian vectors, this rotation can be written compactly as:

$$
\delta \mathbf{r}^{t, r o t}=\overline{\mathbf{R}}_{i n c}^{t,(r)} \delta \mathbf{r}^{t}
$$

where $\overline{\mathbf{R}}_{\text {inc }}^{t}$ stands for the rotation matrix representation of the mean incremental rotation $\overline{\mathbf{q}}_{\text {inc }}^{t(r)}$. Then, the second moments appearing in expression (27) are given by:

$$
\begin{aligned}
& \left\langle\delta \mathbf{r}^{t, r o t} \otimes \delta \mathbf{r}^{t, r o t}\right\rangle^{(r)}=\overline{\mathbf{R}}_{\text {inc }}^{t,(r)}\left\langle\delta \mathbf{r}^{t} \otimes \delta \mathbf{r}^{t}\right\rangle^{(r)} \overline{\mathbf{R}}_{i n c}^{t,(r) T} \\
& \left\langle\delta \mathbf{r}^{t, r o t} \otimes \delta \mathbf{r}_{i n c}^{t}\right\rangle^{(r)}=\overline{\mathbf{R}}_{\text {inc }}^{t,(r)}\left\langle\delta \mathbf{r}^{t} \otimes \delta \mathbf{r}_{i n c}^{t}\right\rangle^{(r)}
\end{aligned}
$$

In Eq. (27), the rotated second moment of misorientation at time $t,\left\langle\delta \mathbf{r}^{t, r o t} \otimes \delta \mathbf{r}^{t, r o t}\right\rangle^{(r)}$, is calculated by rotating the available second moments of misorientation at time $t$ by the mean increment in rotation, while the second moment of misorientation increments, $\left\langle\delta \mathbf{r}_{\text {inc }}^{t} \otimes \delta \mathbf{r}_{\text {inc }}^{t}\right\rangle^{(r)}$, can be calculated based on the first and second moments of the spin, $\langle\dot{\boldsymbol{\omega}}\rangle^{(r)}$ and $\langle\dot{\boldsymbol{\omega}} \otimes \dot{\boldsymbol{\omega}}\rangle^{(r)}$ (see Appendix B). It is important to note that these second moments are already centered and thus are equivalent to covariance matrices [38].

The term $\left\langle\delta \mathbf{r}^{t, r o t} \otimes \delta \mathbf{r}_{i n c}^{t}\right\rangle^{(r)}$ represents the cross-covariance between misorientations and misorientation increments at time $t$, and it is unknown at this point. We propose an approximation of cross-covariance term $\left\langle\delta \mathbf{r}^{t, r o t} \otimes \delta \mathbf{r}_{i n c}{ }^{t}\right\rangle^{(r)}$ based on full-field VPFFT simulations for a face-centered cubic (FCC) polycrystal under tension assuming $\{111\}\langle 1 \overline{1} 0\rangle$ slip, a viscoplastic exponent of $n=10$, and no strain hardening. Thirty different Voronoi polycrystals of 400 grains initialized with exactly the same 400 random orientations, deformed in tension to a strain of $24 \%$, were simulated for this purpose.

The following calculations are performed at three different strain levels $(2 \%, 13 \%$ and $24 \%$ tensile strain) for each grain within each realization of the Voronoi polycrystal. We begin by calculating the eigenvectors $\mathbf{v}^{i, t, r o t}$ and $\mathbf{v}_{\text {inc }}^{i, t}$ and eigenvalues $\lambda^{i, t, r o t}$ and $\lambda_{\text {inc }}^{i, t}$ of $\left\langle\delta \mathbf{r}^{t, r o t} \otimes \delta \mathbf{r}^{t, r o t}\right\rangle^{(r)}$ 
and $\left\langle\delta \mathbf{r}_{\text {inc }}^{t} \otimes \delta \mathbf{r}_{\text {inc }}^{t}\right\rangle^{(r)}$, respectively. In both cases, the eigenvalues essentially represent variances (i.e. the squares of the standard deviations, $S D_{\mathrm{i}}$ ) along corresponding eigenvectors, which, due to the symmetry of the above matrices, form an orthonormal basis of principal axes. The eigenvector corresponding to the largest eigenvalue represents the direction of greatest variation, while the eigenvector corresponding to smallest eigenvalue represents the direction of least variation. The eigenvalues are ordered in descending order and the corresponding eigenvectors are stacked as columns into matrices that transform (note these are not active rotation tensors but coordinate transformation matrices) from principal axes to sample axes: $\mathbf{Q}^{t, r o t}=$ $\left[\mathbf{v}^{1, t, r o t}, \mathbf{v}^{2, t, r o t}, \mathbf{v}^{3, t, r o t}\right]$ and $\mathbf{Q}_{\text {inc }}^{t}=\left[\mathbf{v}_{\text {inc }}^{1, t}, \mathbf{v}_{\text {inc }}^{2, t}, \mathbf{v}_{\text {inc }}^{3, t}\right]$. The cross-covariance term $\left\langle\delta \mathbf{r}^{t, r o t} \otimes \delta \mathbf{r}_{\text {inc }}{ }^{t}\right\rangle$ can now be written as:

$$
\left\langle\delta \mathbf{r}^{t, r o t} \otimes \delta \mathbf{r}_{i n c}^{t}\right\rangle^{(r)}=\mathbf{Q}^{t, r o t}\left\langle\delta \mathbf{r}^{p, t, r o t} \otimes \delta \mathbf{r}_{i n c}^{p, t}\right\rangle^{(r)}\left(\mathbf{Q}_{i n c}^{t}\right)^{T}
$$

where $\delta \mathbf{r}^{p, t, r o t}$ and $\delta \mathbf{r}_{i n c}^{p, t}$ represent the misorientation and the increment in misorientation, respectively, expressed in the corresponding principal axes associated with their respective covariance matrices.

The cross-correlation matrix can then be expressed as:

$$
\left\langle\delta r_{i}^{p, t, r o t}\left(\delta r_{i n c}^{p, t}\right)_{j}\right\rangle^{(r)}=\rho_{i j}^{(r)} \sqrt{\lambda^{i, t, r o t}} \sqrt{\lambda_{\text {inc }}^{j, t}} \text { (no sumation over } i \text { or } j \text { ). }
$$

The cross-correlation coefficient $\rho_{i j}^{(r)}$ represents the strength of linear dependence between variables. The maximal value of $\rho_{i j}^{(r)}$ is 1 and indicates perfect correlation while the minimal value of -1 represents a prefect anti-correlation. Uncorrelated variables will have a correlation coefficient of 0 .

The cross-correlation matrices $\rho_{i j}^{(r)}$ for a grain $r$ of specific orientation were averaged over the thirty different realizations of the Voronoi polycrystals. These matrices turned out to be strongly diagonal after averaging, with fairly similar diagonal values for the entire set of grains. This implies that a strong positive cross-covariance exists between misorientations and increments of misorientation along the corresponding principal directions of largest variation. Analogously, there is also a strong positive cross-covariance between misorientations and increments of misorientation along the corresponding principal directions of medium variation and least variation.

We simplify our analysis even further by averaging cross-correlation matrices over all grains of all orientations and diagonal elements to define the cross-correlation at each strain level by a single value $\rho$. A linear dependence of $\rho$ on the von Mises strain was observed at low strains in the VPFFT simulations and approximated as:

$$
\rho \approx \rho_{0}-\rho_{1} \varepsilon_{V M}
$$


where $\rho_{0}=1$ represents the cross-correlation coefficient at the beginning of deformation and $\rho_{1}$ $=1.3$ represents the strength of the cross-correlation decay with strain (Figure 1).

In the full-field simulations outlined above, a strong correlation between $\delta \mathbf{r}^{t, r o t}$ and $\delta \mathbf{r}_{i n c}^{t}$ is evident. Neglecting the cross-covariance term in the mean-field model would hence result in poor correspondence between the VPFFT and VPSC simulations. With the approximation of the cross-correlation coefficient, $\rho\left(\varepsilon_{V M}\right)$, we can easily calculate the cross-covariance $\left\langle\delta \mathbf{r}^{t, r o t} \otimes \delta \mathbf{r}_{i n c}^{t}\right\rangle^{(r)}$ at any given strain level within the VPSC model.

\subsection{Discrete approximation of misorientations}

For this approximation, we start from the equation for the local orientation update (Eq. 17). As we noted before, orientation, $\mathbf{q}^{t}(\mathbf{x})$, and rotation increment, $\mathbf{q}_{\text {inc }}^{t}(\mathbf{x})$, at time $t$ vary within the grain. Thus, there are certain distributions associated with the orientations and rotation increments at time $t$ within each grain. Consequently, there is a distribution associated with the updated orientations, $\mathbf{q}^{t+\Delta t}(\mathbf{x})$, as well. If we assume each one of these distributions is represented by a discrete set, we obtain a discretized form of Eq. (17):

$$
\mathbf{q}^{t+\Delta t, i}=\mathbf{q}_{\text {inc }}^{t, i} \mathbf{q}^{t, i} ; i=1, n \text { (no summation over } i \text { ). }
$$

The discrete set of $n$ orientations, $\mathbf{q}^{t, i}$, representing the distribution of orientations within the grain at time $t$ is assumed to be known. 27 discrete values within a set are used to describe any distribution assuming that the orientation distribution can be described as multivariate normal distribution (see Appendix C). The discrete set of 27 rotation increments, $\mathbf{q}_{\text {inc }}^{t, i}$, representing the distribution of rotation increments within the grain at time $t$ can be calculated based on the first and second moments of rotation rates, $\langle\dot{\boldsymbol{\omega}}\rangle^{(r)}$ and $\langle\dot{\boldsymbol{\omega}} \otimes \dot{\boldsymbol{\omega}}\rangle^{(r)}$, given by Eq. (11) and (14) in the following way. First, we assume that the distribution associated with the rotation rates, $\dot{\boldsymbol{\omega}}$, is also a multivariate normal distribution. Next, using the first and second moments of rotation rates, we construct a discrete set of values, $\dot{\omega}^{t, i}$, which represents the associated distribution (details are given in Appendix C). Finally, a discrete set of rotation increments is calculated as:

$$
\mathbf{q}_{i n c}^{t, i}=\left\{\begin{array}{c}
\cos \left(\frac{\left|\dot{\boldsymbol{\omega}}^{t, i}\right| \Delta t}{2}\right) \\
\sin \left(\frac{\left|\dot{\boldsymbol{\omega}}^{t, i}\right| \Delta t}{2}\right) \frac{\dot{\boldsymbol{\omega}}^{t, i}}{\left|\dot{\omega}^{t, i}\right|}
\end{array}\right\} .
$$

Once both sets $\mathbf{q}^{t, i}$ and $\mathbf{q}_{\text {inc }}^{t, i}$ are known, the cross-correlation between the rotation increments and orientations needs to be determined. Specifically, we must determine which increment of rotation, $\mathbf{q}_{\text {inc }}^{t, i}$, from the set representing the distribution of rotation increments, corresponds to which orientation, $\mathbf{q}^{t, i}$, from the orientation set. Only then we can calculate the discrete set of $n$ 
updated orientations, $\mathbf{q}^{t+\Delta t, i}$, using Eq. (34). Note that we needed to approximate a similar type of cross-correlation in the previous approach. In what follows, a specific pairing between the elements of two sets $\mathbf{q}^{t, i}$ and $\mathbf{q}_{i n c}^{t, i}$ will be proposed.

The number of possible pairings between two sets $\mathbf{q}^{t, i}$ and $\mathbf{q}_{\text {inc }}^{t, i}$ with $n$ elements is $n$ !. Each one of these pairings corresponds to a different cross-correlation between the increments in rotation and the orientations. If we approximate the cross-correlation based on the results of the VPFFT simulations (as we did for the Taylor expansion approximation) then we have to find the pairing that would result in the resolved cross-correlation. This becomes a difficult task when we consider the number of possible pairings for $n=27$.

In order to choose a paring between sets $\mathbf{q}^{t, i}$ and $\mathbf{q}_{\text {inc }}^{t, i}$ we make the following assumption: if the orientation of a point within the grain reorients in a certain way during the current increment, then that same point will reorient in a similar manner in the next increment. This implies that a strong positive cross-correlation exists between increments in rotation from two subsequent time steps $\left(\mathbf{q}_{\text {inc }}^{t, i}\right.$ and $\left.\mathbf{q}_{\text {inc }}^{t-\Delta t, i}\right)$. Therefore, we adopt such pairing between discrete increments in rotation from current, $\mathbf{q}_{\text {inc }}^{t, i}$, and previous increment, $\mathbf{q}_{\text {inc }}^{t-\Delta t, i}$, that results in the strongest possible positive cross-correlation. Finding such a pairing is not difficult, since these two sets are quite similar considering that they correspond to two subsequent increments. Consequently, we have also defined the pairing between $\mathbf{q}_{\text {inc }}^{t, i}$ and $\mathbf{q}^{t, i}$, since the pairing between $\mathbf{q}^{t-\Delta t, i}$ inc and $\mathbf{q}^{t, i}$ is available from the previous increment. Therefore, the achieved correlation between the rotation increments, $\mathbf{q}_{\text {inc }}^{t, i}$, and orientations, $\mathbf{q}^{t, i}$, is a consequence of the assumed correlation between rotation increments from two subsequent steps $\mathbf{q}_{\text {inc }}^{t, i}$ and $\mathbf{q}_{\text {inc }}^{t-\Delta t, i}$.

Once the updated set of orientations $\mathbf{q}^{t+\Delta t, i}$ is obtained, the mean orientation, misorientations with respect to the mean orientation, and their second moments can be easily calculated [38]. In the discrete approach the updating of orientations is done in the proper way (Eq. (34)), while in the Taylor expansion approach we used a first order Taylor approximation (Eq. (21)). Certain errors will come from the fact that all of the distributions are represented with only a finite number of points ( 27 in our simulations). However, the biggest issue of the discrete approach is related to the fact that the cross-correlation of rotation increments between two subsequent time steps is always assumed as the strongest possible. Consequently, we should expect to obtain large orientation spreads along largest principal direction.

In both approaches the intragranular stress distributions drive the development of intragranular misorientation. On the other hand, in the previous attempts for prediction of intragranular misorientation implemented within VPSC, different reorientations of grains from co-rotation with their respective neighbors was considered as driving force for the misorientation evolution, interpreting the developing intergranular misorientation distribution between grains of same initial orientation simply as intragrain misorientation distribution of grains with this initial 
orientation $[20,22]$. As the reorientation is completely determined by the grains neighborhood defined usually in a random manner, the predicted misorientation distributions were virtually independent of the particular grain orientation and unrelated to existing intragranular distributions of micromechanical fields.

\section{Results and discussion}

In what follows, we exercise the two developed models on two FCC materials and compare the results to experiments and simulation results obtained by the full-field VPFFT model [11, 39]. In all the simulations, it is assumed that the grains deform by $\{111\}\langle 1 \overline{1} 0\rangle$ slip with a viscoplastic power-law exponent of $n=10$ without any strain hardening. For every grain, the mean orientation and second order moment of misorientation distribution are calculated as described above. The Taylor expansion approximation provides these quantities as an output. The dominant axis of rotation is defined as the principal direction of misorientation covariance matrix corresponding to the largest standard deviation $[38,40]$. As a measure of the magnitude of the misorientation spread, we use the equivalent isotropic spread calculated as the geometrical mean of the three standard deviations $S D=\sqrt[3]{S D 1 \times S D 2 \times S D 3}$ [41]. The specific case studies considered are uniaxial tension of $\mathrm{Cu}$ and plane-strain compression of $\mathrm{Al}$.

\subsection{Tension of polycrystalline copper}

We simulate tension of polycrystalline $\mathrm{Cu}$ to a strain of $11 \%$ with the VPSC model accounting for intragranular misorientation evolution using the Taylor expansion approximation and the discrete approximation of misorientations, on the one hand; and with VPFFT, on the other hand. The initial texture of the recrystallized $\mathrm{Cu}$ sample is represented by 400 grains (Figure 2). These grains are assumed to be initially spherical for the VPSC simulations and to be arranged in a Voronoi tessalation microstructural cell in case of the VPFFT model. Predictions of the intragranular misorientation distributions are compared with experimental results published in [39].

For the experimental results obtained by EBSD, only grains with more than 350 indexed scan points (corresponding to an apparent area larger than $1400 \mu \mathrm{m}^{2}$ ) are considered, to facilitate comparisons. Small grains in two-dimensional EBSD scans could correspond to sections close to the center of mass of small grains within the sample or they could represent sections far away from the center of mass of larger grains. In the former case, the obtained misorientation distribution might not be representative, since the deformation within small grains might be influenced greatly by its larger neighbors [39]. In the latter case, the misorientation distribution is not representative since we have sampled only small and localized number of points within a large grain. In addition, the evolution in small parts of a large grain is also affected by the neighbors.

The shapes of the misorientation distributions are classified into three categories (see Table 1) according to the ratios of the standard deviations along principal directions. This classification is 
a variation of the one proposed by [41] with the classes "ellipsoid" and "prolate" merged into a single "prolate" category. Figure 3 compares the dependence between shapes of misorientation distributions and orientation for all of the considered cases. Whereas EBSD and VPFFT results show variations of the shape for similar orientations, VPSC subdivides the triangle into certain regions preferring a singular type. This is expected since in VPSC there is no neighborhood effect and behavior of every grain is defined solely by its orientation. Table 2 indicates that fractions of different distribution shapes are similar between all cases. In the EBSD and VPFFT results, spherical shapes develop in regions close to [111] and [001], while in VPSC spherical shapes concentrate only around [111]. Prolate shapes seem to be spread out over the entire IPF in the EBSD and VPFFT results. VPSC predicts relatively similar trends but with special regions, where no prolates are found. Oblate shapes also seem to be distributed evenly for the EBSD and VPFFT results, while VPSC predictions of oblates are concentrated around [111], [001] and [114] to [112].

Figure 4 compares the direction of the dominant rotation axes of grains with oblate and prolate misorientation distribution shapes plotted in the sample frame. The dominant rotation axes predominantly lie in a plane perpendicular to the tension direction in the simulation results. On the other hand, the experimental results show a considerably weaker trend of the dominant rotation axes to align perpendicular to the tension direction, with much more scatter.

The evolution of misorientation spread is determined by the plastic spin distribution which is in turn caused by the stress distribution within the grain. We can examine the sensitivity of the plastic spin components to fluctuations in stress by calculating the derivatives of plastic spin with respect to stress [42]:

$$
\frac{\partial \dot{\boldsymbol{\omega}}^{p}}{\partial \boldsymbol{\sigma}}=\sum_{S} \frac{\partial \dot{\gamma}^{s}}{\partial \tau^{s}} \boldsymbol{\alpha}^{s} \otimes \mathbf{m}^{s} .
$$

These calculations are performed using the VPSC model. The stress tensor and the symmetric Schmid tensor are represented as five dimensional vectors of the form [43]: $\boldsymbol{\sigma}=\sqrt{2}$ $\left(\frac{\left(\sigma_{33}-\sigma_{11}\right)+\left(\sigma_{33}-\sigma_{22}\right)}{2 \sqrt{3}}, \frac{\sigma_{22}-\sigma_{11}}{2}, \sigma_{23}, \sigma_{13}, \sigma_{12}\right)$. The derivative of $i$-th component of plastic spin with respect to stress vector, $\frac{\partial \dot{\omega}_{i}^{p}}{\partial \boldsymbol{\sigma}}$, represents a gradient vector in stress space, which defines the rate of change of $i$-th plastic spin component with respect to changes in stress. By examining the gradient vectors of each plastic spin component within the grains we find that the length of the gradient vector of the plastic spin component along the tension direction is considerably smaller than the lengths of the other two gradient vectors. Therefore, the plastic spin component in the tension direction is less sensitive to the fluctuations in stress than the other two components. Consequently, for isotropic fluctuations in stress, the fluctuations in plastic spin will have only a small component in the tension direction which in turn causes the dominant rotation axes to lie in the plane perpendicular to the tension direction. In addition, an anisotropic shape of the stress distribution also affects the plastic spin distribution. However, the fact that the dominant rotation 
axes are perpendicular to the tension direction in the simulations is predominantly caused by the insensitivity of plastic spin component in the tension direction to fluctuations in stress.

Figure 5 shows the predicted and experimental probability density functions (pdf) of the standard deviations along the principal directions and the equivalent isotropic spreads. The pdfs for the first two standard deviations match quite well for all considered cases. Both, VPFFT and experimental pdfs of the smallest standard deviation display a single characteristic peak around 0.01. On the other hand, the VPSC pdfs of the smallest standard deviation develop an additional peak close to 0 indicating that the spread is almost two-dimensional within a certain number of grains. The occasionally small magnitude of the third standard deviation significantly reduces the magnitude of the corresponding equivalent isotropic spread and therefore the VPSC predictions for the pdfs of the equivalent isotropic spread also develop two peaks while corresponding pdfs from the VPFFT simulations and the experiment develop only one. In addition, we have also performed simulations up to a strain of $25 \%$ and compared the misorientation distribution shapes with experimental results published in [41] (Table 3). Prolate shapes dominate the region near [011] and other regions away from [001] or [111] both in the simulations and experimental results. According to Dillamore and Katoh (Dillamore and Katoh, 1974), grains with tensile axis on the line connecting [114] and [011] show divergent re-orientations and large orientation spreads within them are expected to develop along a particular axis. Due to such a strong orientation spread developing around a preferred axis, grains from divergent regions should develop prolate shapes of misorientation distribution which is in agreement with both simulation results and experiment (see the region near [110] and the remaining region away from [011] and [111] in Table 3). The regions near [001] and [111] are convergent and hence should develop lower orientation spreads [2]. While the experimental results reveal a rather high fraction of prolates above $50 \%$ even for these regions, the predictions of the simulations are significantly lower. In particular, a quite low fraction of prolates is expected from the simulations for the [111] region.

In summary, we observe a reasonable agreement between the simulations and the experimental results, with some peculiar differences between VPFFT and VPSC, but no significant difference between the two developed VPSC models for updating of misorientation spreads at lower strain levels in FCC metals.

\subsection{Plane-strain compression of polycrystalline aluminum}

In addition to uniaxial deformation, we also simulate plane-strain compression of fcc polycrystal to $38 \%$ reduction and compare the predictions to results obtained after cold-rolling of aluminum [44]. The initial texture was represented by 400 randomly oriented grains of equal volume fraction.

The orientations of the dominant rotation axes are shown in Fig. 6. The dominant rotation axes are predominantly aligned with the transverse direction in both, simulations and experiment. 
Clustering of the dominant rotation axes around the transverse direction is considerably stronger in the VPSC predictions. Similar trends were observed for hot rolled aluminum at lower reductions [42].

Figure 7 shows the probability density functions of the equivalent isotropic spreads which all show only a single peak and no second peak at lower equivalent isotropic spreads is observed. It is seen that the simulations overpredict the magnitudes of the equivalent isotropic spread. The discrete approximation approach displays the largest values for the equivalent isotropic spread, since in this model a strong correlation between subsequent misorientation increments is enforced. This corroborates the statement that the main difference between the two approaches lies in the approximation of the cross-correlation term. The simulated equivalent orientation spreads are in all cases too large in comparison to the experimental results. This discrepancy can be attributed to a number of reasons. At higher strains, certain grains in the VPFFT simulation will develop a quite large misorientation spread along the dominant rotation axis. Closer inspection reveals that the sign-carrying misorientation distribution along this direction is actually bimodal, which indicates that grain has fragmented into two parts [45, 46]. By not considering the fragmented grains separately we have indirectly increased the values of equivalent isotropic spread. Similarly, in a VPSC simulation some grains develop very large spreads in a certain direction indicating that they should be fragmented and considered separately. In addition, the threshold angle used for identification of individual grains in the orientation maps was $8^{\circ}$ which also affects the evaluation of the experimental results. During deformation, deformation-induced boundaries are forming within the grains causing their subdivision [47]. Some of these boundaries will have misorientation angles greater than $8^{\circ}$. Therefore, fragments of initially one grain will be treated as separate grains in the experimental results, when entirely separated by such a deformation-induced boundary with an angle above $8^{\circ}$. This procedure of treating fragments individually as seperate grains reduces, in general, the magnitude of the misorientation spread within the grains.

The predicted intragranular misorientation spreads obtained here show the need for further development of the VPSC model to include grain fragmentation towards more accurate meanfield simulations of deformation and recrystallization texture evolution. As noted above, the grains with largest standard deviations in VPFFT results develop bimodal sign-carrying misorientation distributions along the dominant rotation axis. Analogously, we could assume that the grains with the largest standard deviations in the VPSC model go through a similar type of fragmentation. The parent grain would be divided into two children grains whose mean orientations and misorientation spreads would be defined based on the mean orientation and the anisotropic misorientation spread of the parent, for instance by replacing the parent grain by two children having a misorientation between their mean orientations along the dominant misorientation axis. The misorientation spreads could also be used for identifying grains that have a tendency to develop transition bands which is a prerequisite for modeling of recrystallization dominated by nucleation at transition bands. Even in cases where the nucleation 
occurs at existing grain boundaries, the information about the misorientation spreads could improve recrystallization texture predictions.

\section{Conclusions}

In this work, we have extended existing algorithms to obtain second moments of the micromechanical fields already implemented in VPSC to include the calculation of intragranular misorientations. Two approaches were presented for updating the intragranular misorientation distributions. It was found that the Taylor expansion approach was simpler, required less calculations and realized more freedom in the approximation of the cross-correlation term between misorientations and rotation-rates than the discrete approximation. The predictive characteristics of the proposed models were evaluated on the deformation of two FCC polycrystalline materials $(\mathrm{Cu}$ deformed in tension and $\mathrm{Al}$ deformed in plane-strain compression/rolling), and compared with corresponding experiments and appropriate full-field VPFFT simulation results. Acceptable predictions of orientation spreads were achieved using VPSC, with tremendous computational advantages over full-field models (by approximately three orders of magnitude). It was observed that at higher strains, some of the grains develop quite large misorientation spreads suggesting fragmentation into individual parts. Development of models for grain fragmentation and recrystallization taking advantage of the proposed algorithms, and extensions for metals other than FCC such as hexagonal metals will be pursued in future efforts.

\section{Appendix A}

Unit quaternion representation of rotations

Rotations can be represented as unit quaternions in the following form:

$$
\mathbf{q}=\left\{\begin{array}{c}
\cos \frac{\theta}{2} \\
n_{i} \sin \frac{\theta}{2}
\end{array}\right\}=\left\{\begin{array}{c}
\cos \frac{\theta}{2} \\
r_{i}
\end{array}\right\}=\left\{\begin{array}{l}
a \\
b \\
c \\
d
\end{array}\right\}
$$

where $n_{i}$ and $\theta$ represent rotation axis and angle, respectively. The vector part of the unit quaternion $r_{i}$ is defined as $r_{i}=n_{i} \sin \frac{\theta}{2}$. There are several different matrix representations of unit quaternions, we have adopted the following [36]:

$$
\mathbf{q}=\left[\begin{array}{cccc}
a & c & b & d \\
-c & a & d & -b \\
-b & -d & a & c \\
-d & b & -c & a
\end{array}\right]
$$


Consequently, we can deal with matrices and matrix algebra instead of quaternion algebra. Product of two unit quaternions can then be expressed as simple matrix multiplication:

$$
q_{i j}=q_{i k}^{A} q_{k j}^{B}
$$

The matrix representation of unit quaternions was used only in derivations of certain equations. The actual implementation of final equations was not done in matrix form due to computational inefficiency of this formulation.

\section{Appendix B}

\section{Incremental rotations}

Incremental rotations and their first and second moments can be directly calculated from first and second moments of spin. First we define incremental rotation at material point using small angle approximation:

$$
\mathbf{q}_{\text {inc }}(\mathbf{x})=\left\{\begin{array}{c}
\cos \frac{\Delta \theta(\mathbf{x})}{2} \\
\sin \frac{\Delta \theta(\mathbf{x})}{2} \mathbf{n}(\mathbf{x})
\end{array}\right\} \approx\left\{\begin{array}{c}
1-\frac{1}{8} \Delta \theta^{2}(\mathbf{x}) \\
\frac{1}{2} \Delta \theta(\mathbf{x}) \mathbf{n}(\mathbf{x})
\end{array}\right\}
$$

First moment of incremental rotation is:

$$
\left\langle\mathbf{q}_{\text {inc }}\right\rangle=\left\{\begin{array}{c}
1-\frac{1}{8}\left\langle\Delta \theta^{2}\right\rangle \\
\frac{1}{2}\langle\Delta \theta \mathbf{n}\rangle
\end{array}\right\}
$$

The mean incremental rotation can then be calculated as: $\overline{\mathbf{q}}_{\text {inc }}=\left\langle\mathbf{q}_{\text {inc }}\right\rangle /\left|\left\langle\mathbf{q}_{\text {inc }}\right\rangle\right|$. The dyadic product of incremental rotations can be written as:

$$
\mathbf{q}_{i n c}(\mathbf{x}) \otimes \mathbf{q}_{i n c}(\mathbf{x})=\left[\begin{array}{cc}
1-\frac{1}{4} \Delta \theta^{2}(\mathbf{x}) & \frac{1}{2} \Delta \theta(\mathbf{x}) \mathbf{n}^{T}(\mathbf{x}) \\
- \text { symmetric }- & \frac{1}{4} \Delta \theta^{2}(\mathbf{x}) \mathbf{n}(\mathbf{x}) \otimes \mathbf{n}(\mathbf{x})
\end{array}\right]+\mathrm{O}\left(\Delta \theta^{3}(\mathbf{x})\right)
$$

The second moment is then simply:

$$
\left\langle\mathbf{q}_{\text {inc }} \otimes \mathbf{q}_{\text {inc }}\right\rangle=\left[\begin{array}{cc}
1-\frac{1}{4}\left\langle\Delta \theta^{2}\right\rangle & \frac{1}{2}\left\langle\Delta \theta \mathbf{n}^{T}\right\rangle \\
- \text { symmetric }- & \frac{1}{4}\left\langle\Delta \theta^{2} \mathbf{n} \otimes \mathbf{n}\right\rangle
\end{array}\right]
$$

After substitution of spin into the above expression we get: 


$$
\begin{aligned}
& \left\langle\mathbf{q}_{\text {inc }}\right\rangle=\left\{\begin{array}{c}
1-\frac{1}{8} \mathbf{I}:\langle\dot{\boldsymbol{\omega}} \otimes \dot{\boldsymbol{\omega}}\rangle \Delta t^{2} \\
\frac{1}{2}\langle\dot{\boldsymbol{\omega}}\rangle \Delta t
\end{array}\right\} \\
& \left\langle\mathbf{q}_{\text {inc }} \otimes \mathbf{q}_{\text {inc }}\right\rangle=\left[\begin{array}{cc}
1-\frac{1}{4} \mathbf{I}:\langle\dot{\boldsymbol{\omega}} \otimes \dot{\boldsymbol{\omega}}\rangle \Delta t^{2} & \frac{1}{2}\langle\dot{\boldsymbol{\omega}}\rangle^{T} \Delta t \\
- \text { symmetric }- & \frac{1}{4}\langle\dot{\boldsymbol{\omega}} \otimes \dot{\boldsymbol{\omega}}\rangle \Delta t^{2}
\end{array}\right]
\end{aligned}
$$

The second moment of misorientation $\left\langle\delta \mathbf{q}_{\text {inc }} \otimes \delta \mathbf{q}_{\text {inc }}\right\rangle$ can be calculated using the first and second moments of total increments in rotation.

\section{Appendix C}

\section{Discrete representation of multivariate distribution}

The spin distribution is defined by first, $\langle\dot{\boldsymbol{\omega}}\rangle$, and second, $\langle\dot{\boldsymbol{\omega}} \otimes \dot{\boldsymbol{\omega}}\rangle$, moments from which covariance matrix can be calculated as $\langle\dot{\boldsymbol{\omega}} \otimes \dot{\boldsymbol{\omega}}\rangle-\langle\dot{\boldsymbol{\omega}}\rangle \otimes\langle\dot{\boldsymbol{\omega}}\rangle$. We shall assume that the distribution associated with the spin field within the phase $r$ is multivariate normal distribution.

First, the principal values and directions of covariance matrix are calculated. The multivariate normal distribution implies that distributions associated with the principal directions are univariate normal. Next each one of these univariate distributions is approximated by certain number, $m$, of discrete values with equal probability [48]. In all of the simulations we have used three $(m=3)$ points along each principal direction, one as a mean and the other two were symmetric for + and - angle.

Next the approximation of multivariate distribution is constructed from discrete univariate distributions as follows. In the principal frame of covariance matrix there is no correlation between different components of spin vector:

$$
\left\langle\dot{\omega}_{i}^{p} \dot{\omega}_{j}^{p}\right\rangle-\left\langle\dot{\omega}_{i}^{p}\right\rangle \otimes\left\langle\dot{\omega}_{j}^{p}\right\rangle=0, \text { for } i \neq j
$$

where superscript $p$ denotes a variable expressed in the principal frame. In the case of multivariate normal distribution this implies independence of the spin components. The discrete approximation of the multivariate normal distribution is then defined by combining the discrete values of the univariate distributions associated with each spin component in principal frame, giving in total $n=m^{3}$ discrete vectors (27 in our simulations). Finally, we transfer the spin vectors to the global frame and obtain a discrete representation of the spin distribution given by a set of $n$ discrete values $\dot{\boldsymbol{\omega}}^{i}$. 


\section{Acknowledgments}

M. Z. and M. K. acknowledge subcontract, No. 388715, granted by Los Alamos National Laboratory to the University of New Hampshire. M. Z. also acknowledges the Seaborg Research Fellowship and wishes to thank Andrew Richards for numerous discussions and suggestions. The work at Los Alamos National Laboratory is supported by the Los Alamos National Laboratory Directed Research and Development (LDRD) project 20140630ER. Los Alamos National Laboratory is operated by Los Alamos National Security LLC under DOE Contract DEAC52-06NA25396.

\section{References}

[1] W.F. Hosford. The Mechanics of Crystals and Textured Polycrystals, Oxford University Press, USA, 1993.

[2] I. Dillamore, H. Katoh. Mechanisms of recrystallization in cubic metals with particular reference to their orientation-dependence., Metal Science 8 (1974) 73-83.

[3] F. Humphreys, M. Hatherly. Recrystallization and related annealing phenomena, 2004. Elsevier, 2004.

[4] R.J. McCabe, A.W. Richards, D.R. Coughlin, K.D. Clarke, I.J. Beyerlein, M. Knezevic. Microstructure effects on the recrystallization of low-symmetry alpha-uranium, Journal of Nuclear Materials 465 (2015) 189-195.

[5] S.R. Kalidindi, C.A. Bronkhorst, L. Anand. Crystallographic texture evolution in bulk deformation processing of FCC metals, J. Mech. Phys. Solids 40 (1992) 537-569.

[6] M. Knezevic, B. Drach, M. Ardeljan, I.J. Beyerlein. Three dimensional predictions of grain scale plasticity and grain boundaries using crystal plasticity finite element models, Computer Methods in Applied Mechanics and Engineering 277 (2014) 239-259.

[7] M. Zecevic, R.J. McCabe, M. Knezevic. A new implementation of the spectral crystal plasticity framework in implicit finite elements, Mechanics of Materials 84 (2015) 114-126.

[8] M. Ardeljan, I.J. Beyerlein, M. Knezevic. A dislocation density based crystal plasticity finite element model: Application to a two-phase polycrystalline HCP/BCC composites, J. Mech. Phys. Solids 66 (2014) 16-31.

[9] M. Zecevic, R.J. McCabe, M. Knezevic. Spectral database solutions to elastoviscoplasticity within finite elements: Application to a cobalt-based FCC superalloy, Int. J. Plast. 70 (2015) 151-165.

[10] H. Moulinec, P. Suquet. A numerical method for computing the overall response of nonlinear composites with complex microstructure, Computer methods in applied mechanics and engineering 157 (1998) 69-94.

[11] R.A. Lebensohn. N-site modeling of a 3D viscoplastic polycrystal using Fast Fourier Transform, Acta Materialia 49 (2001) 2723-2737.

[12] R. Hill. Continuum micro-mechanics of elastoplastic polycrystals, Journal of the Mechanics and Physics of Solids 13 (1965) 89-101.

[13] J.W. Hutchinson. Proceedings of the Royal Society of London A319 (1970) 247.

[14] A. Molinari, G.R. Canova, S. Ahzi. Self consistent approach of the large deformation polycrystal viscoplasticity, Acta Metall. Mater. 35 (1987) 2983-2994. 
[15] R.A. Lebensohn, C.N. Tomé. A self-consistent anisotropic approach for the simulation of plastic deformation and texture development of polycrystals: Application to zirconium alloys, Acta Metall. Mater. 41 (1993) 2611-2624.

[16] S. Nemat-Nasser, M. Hori. Micromechanics: overall properties of heterogeneous materials, Elsevier, 2013.

[17] P. Ponte Castañeda. Second-order homogenization estimates for nonlinear composites incorporating field fluctuations: I-theory, Journal of the Mechanics and Physics of Solids 50 (2002) 737-757.

[18] Y. Liu, P.P. Castañeda. Second-order theory for the effective behavior and field fluctuations in viscoplastic polycrystals, Journal of the Mechanics and Physics of Solids 52 (2004) 467-495.

[19] M. Berveiller, H. Bouaouine, N. Fakri, P. Lipinski. Texture Transition, Micro Shear Bands and Heterogeneous Plastic Strain in FCC and BCC Metals in Rolling, Texture, Stress, and Microstructure 8 (1988) 351-379.

[20] R.E. Bolmaro, A. Fourty, A. Roatta, M.A. Bertinetti, P.A. Turner, J.W. Signorelli. A new approach to crystal spin calculation during deformation texture development, Scripta Materialia 43 (2000) 553-559.

[21] R.E. Bolmaro, A. Roatta, A.L. Fourty, J.W. Signorelli. Recrystallization textures in fcc materials: A simulation based on micromechanical modeling data, Scripta Materialia 53 (2005) 147-152.

[22] C. Tomé, C. Necker, R. Lebensohn. Mechanical anisotropy and grain interaction in recrystallized aluminum, Metall. Mater. Trans. A 33 (2002) 2635-2648.

[23] L.S. Toth, Y. Estrin, R. Lapovok, C.F. Gu. A model of grain fragmentation based on lattice curvature, Acta Materialia 58 (2010) 1782-1794.

[24] D. Raabe. Simulation of texture evolution during rolling deformation of an intermetallic Fe-28Al-5Cr polycrystal, Materials Letters 19 (1994) 75-78.

[25] D. Raabe, Z. Zhao, S.-J. Park, F. Roters. Theory of orientation gradients in plastically strained crystals, Acta Materialia 50 (2002) 421-440.

[26] C.S. Lee, B.J. Duggan. Deformation banding and copper-type rolling textures, Acta Metall. Mater. 41 (1993) 2691-2699.

[27] T. Leffers. A model for rolling deformation with grain subdivision. Part I: The initial stage, International Journal of Plasticity 17 (2001) 469-489.

[28] G.C. Butler, D.L. McDowell. Polycrystal constraint and grain subdivision, International Journal of Plasticity 14 (1998) 703-717.

[29] X. Guo, M. Seefeldt. Modeling grain fragmentation and deformation textures for titanium using a combined approach of the viscoplastic self-consistent model and a shear fluctuation model, Journal of Materials Science 52 (2017) 8132-8148.

[30] R.A. Lebensohn, Y. Liu, P.P. Castaneda. On the accuracy of the self-consistent approximation for polycrystals: comparison with full-field numerical simulations, Acta Materialia 52 (2004) 5347-5361.

[31] R.A. Lebensohn, C.N. Tome, P.P. Castaneda. Self-consistent modelling of the mechanical behaviour of viscoplastic polycrystals incorporating intragranular field fluctuations, Philosophical Magazine 87 (2007) 4287-4322.

[32] R.A. Lebensohn, M. Zecevic, M. Knezevic, R.J. McCabe. Average intragranular misorientation trends in polycrystalline materials predicted by a viscoplastic self-consistent approach, Acta Materialia 104 (2016) 228-236. 
[33] R. Masson, M. Bornert, P. Suquet, A. Zaoui. An affine formulation for the prediction of the effective properties of nonlinear composites and polycrystals, J. Mech. Phys. Solids 48 (2000) 1203-1227.

[34] J.D. Eshelby. The determination of the elastic field of an ellipsoidal inclusion, and related problems, Proc R. Soc. Lond. A 241 (1957) 376-396.

[35] A. Papoulis. Probability, Random Variables and Stochastic Processes. Series in Electrical Engineering: Communications and Signal Processing. New York: McGraw-Hill, Inc., 1991.

[36] R.W. Farebrother, J. Gross, S.O. Troschke. Matrix representation of quaternions, Linear Algebra Appl 362 (2003) 251-255.

[37] A.H.S. Ang, W.H. Tang. Probability Concepts in Engineering Planning and Design, Basic Principles, Wiley, 1975.

[38] W. Pantleon. Retrieving orientation correlations in deformation structures from orientation maps, Materials Science and Technology 21 (2005) 1392-1396.

[39] R.A. Lebensohn, R. Brenner, O. Castelnau, A.D. Rollett. Orientation image-based micromechanical modelling of subgrain texture evolution in polycrystalline copper, Acta Materialia 56 (2008) 3914-3926.

[40] F. Bachmann, R. Hielscher, P.E. Jupp, W. Pantleon, H. Schaeben, E. Wegert. Inferential statistics of electron backscatter diffraction data from within individual crystalline grains, Journal of Applied Crystallography 43 (2010) 1338-1355.

[41] S. Krog-Pedersen, J.R. Bowen, W. Pantleon. Quantitative characterization of the orientation spread within individual grains in copper after tensile deformation, International Journal of Materials Research 100 (2009) 433-438.

[42] R. Quey, J.H. Driver, P.R. Dawson. Intra-grain orientation distributions in hot-deformed aluminium: Orientation dependence and relation to deformation mechanisms, Journal of the Mechanics and Physics of Solids 84 (2015) 506-527.

[43] P. Lequeu, P. Gilormini, F. Montheillet, B. Bacroix, J.J. Jonas. Yield Surfaces for Textured Polycrystals .1. Crystallographic Approach, Acta Metallurgica 35 (1987) 439-451.

[44] W. Pantleon, W. He, T.P. Johansson, C. Gundlach. Orientation inhomogeneities within individual grains in cold-rolled aluminium resolved by electron backscatter diffraction, Mater. Sci. Eng. A-Struct. Mater. Prop. Microstruct. Process. 483-84 (2008) 668-671.

[45] T.E. Buchheit, J.D. Carroll, B.G. Clark, B.L. Boyce. Evaluating Deformation-Induced Grain Orientation Change in a Polycrystal During In Situ Tensile Deformation using EBSD, Microsc Microanal 21 (2015) 969-984.

[46] R. Quey, P.R. Dawson, J.H. Driver. Grain orientation fragmentation in hot-deformed aluminium: Experiment and simulation, Journal of the Mechanics and Physics of Solids 60 (2012) 509-524.

[47] D.A. Hughes, N. Hansen. High angle boundaries formed by grain subdivision mechanisms, Acta. Mater. 45 (1997) 3871-3886.

[48] A.C. Miller, T.R. Rice. Discrete Approximations of Probability-Distributions, Manage Sci 29 (1983) 352-362. 


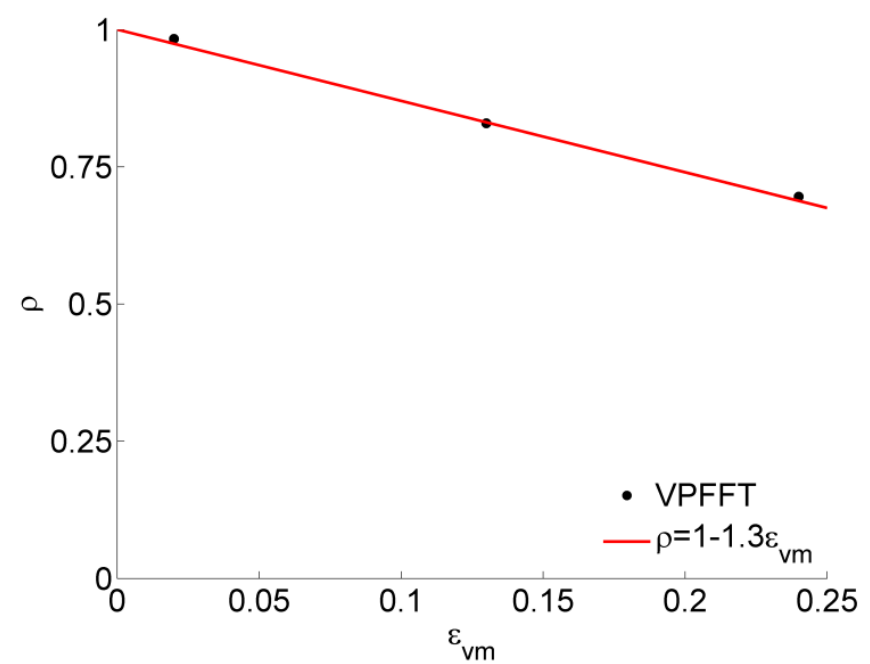

Figure 1. Correlation parameter $\rho$ derived from VPFFT simulation of 30 realizations of Voronoi polycrystals with 400 grains as an average over all grains at three different strain levels fitted with linear function. 

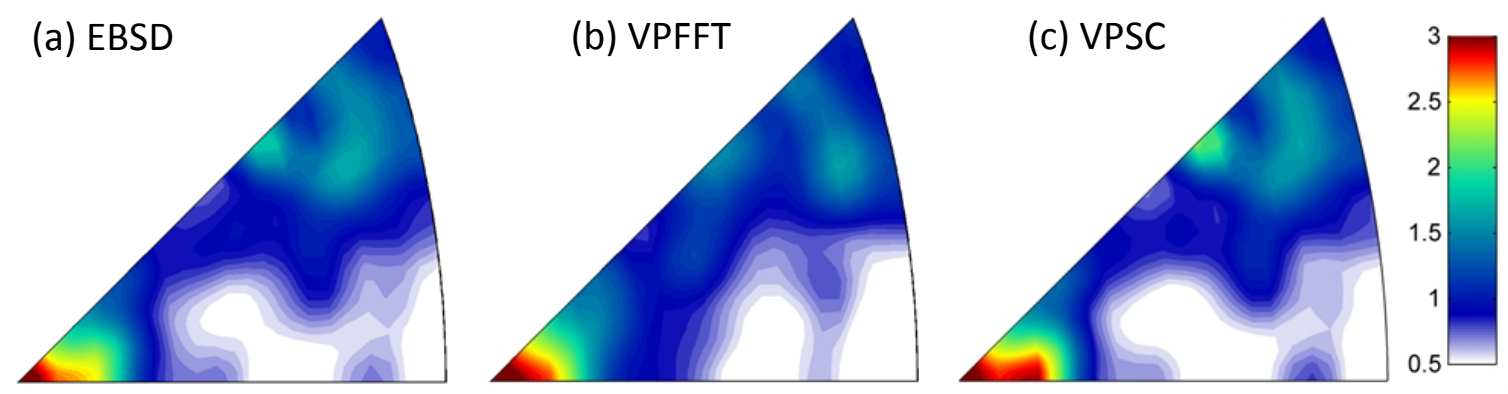

Figure 2. Inverse pole figures for the crystallographic direction along the tensile axis illustrating the measured initial texture (a) and the initial textures used in VPFFT (b) and VPSC (c) simulations. 

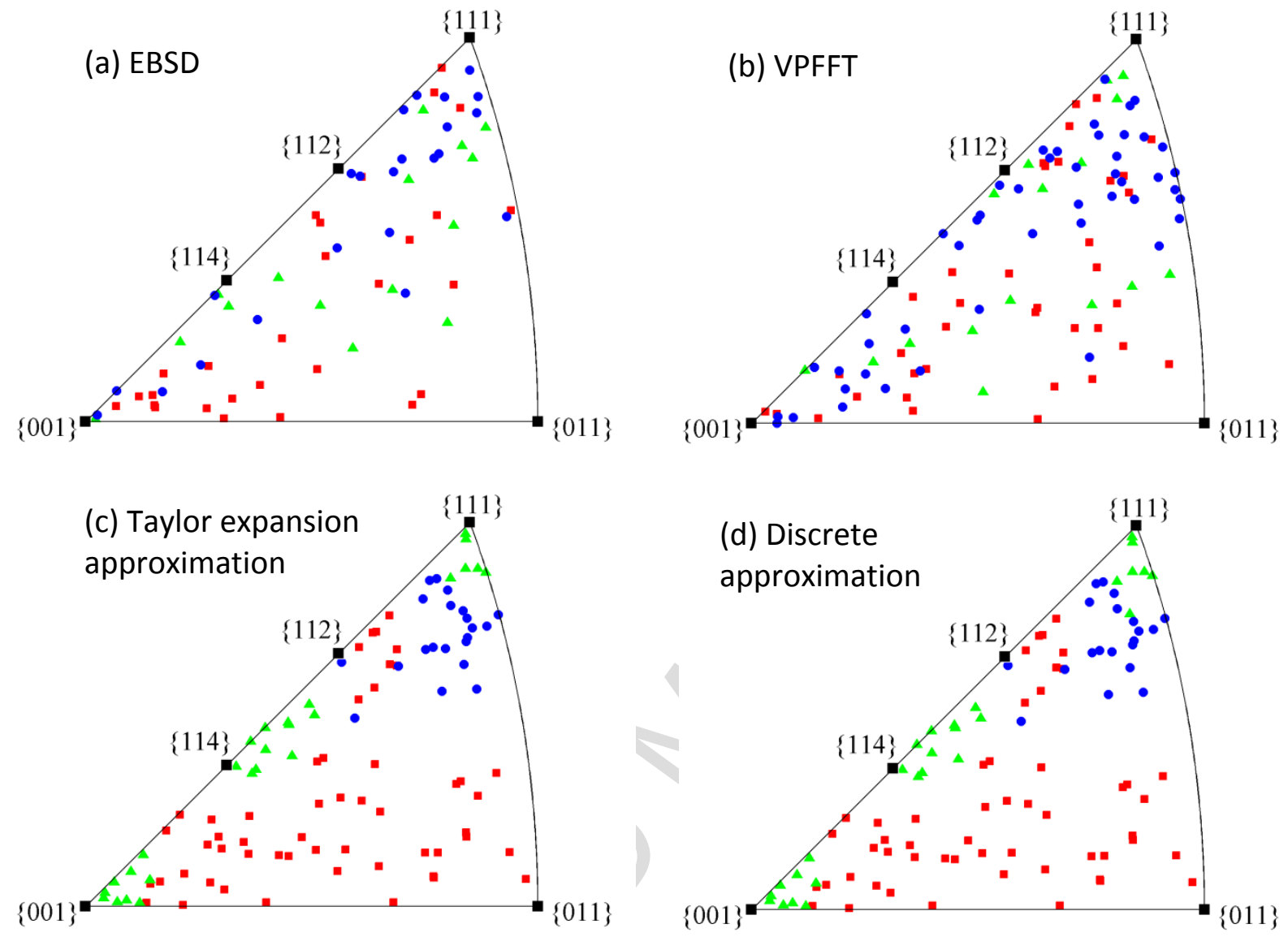

- Spherical - Prolate $\triangle$ Oblate

Figure 3. Shapes of misorientation spreads at $11 \%$ tensile strain categorized according to Table 1 plotted as inverse pole figures for (a) EBSD data, (b) full-field VPFFT results, (c) Taylor expansion approximation and (d) discrete approximation approaches. For better visualization, plots of simulation results are for 100 grains randomly picked from the simulated polycrystals. For the both VPSC simulations the same 100 grains are used. 
(a) EBSD

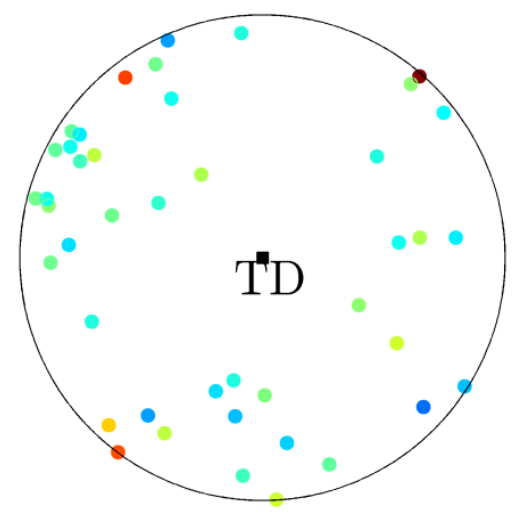

(c) Taylor expansion approx.

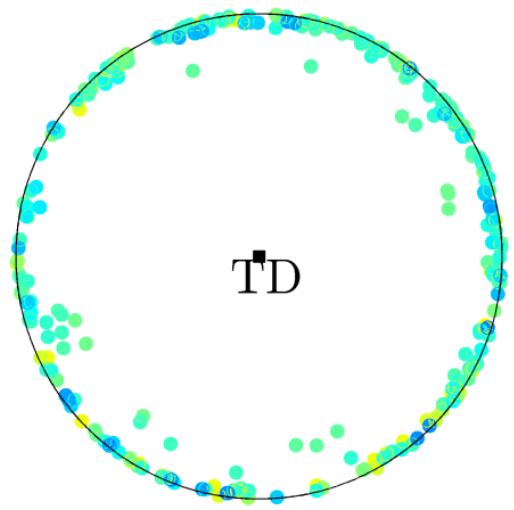

SD1 (b) VPFFT

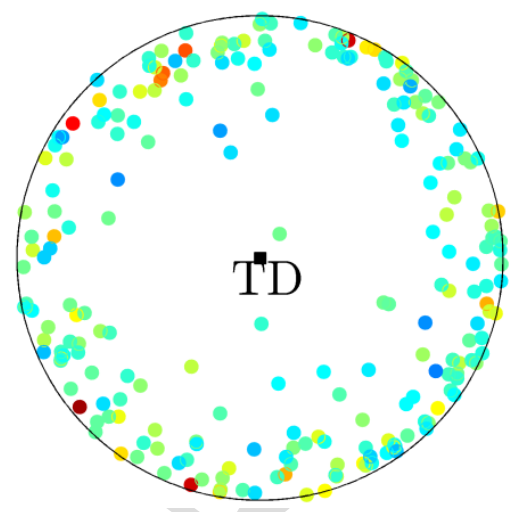

(d) Discrete approx.

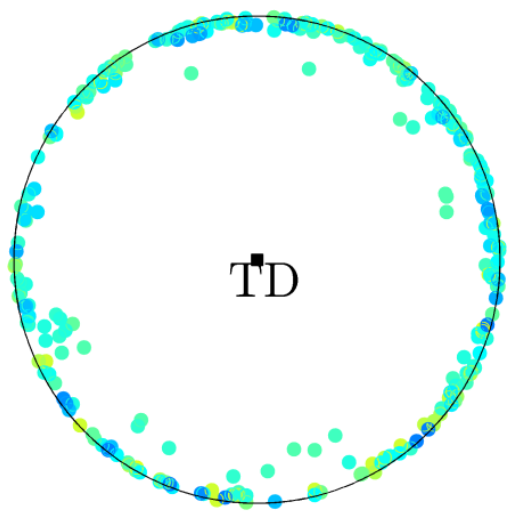

0.04

Figure 4. Dominant rotation axes for oblate and prolate shapes plotted in the sample frame and color-coded according to magnitude of standard deviation along this direction for (a) EBSD data, (b) VPFFT results, (c) Taylor expansion approximation and (d) discrete approximation approach at $11 \%$ tensile strain. Tension direction (TD) is in the center of pole figures. 

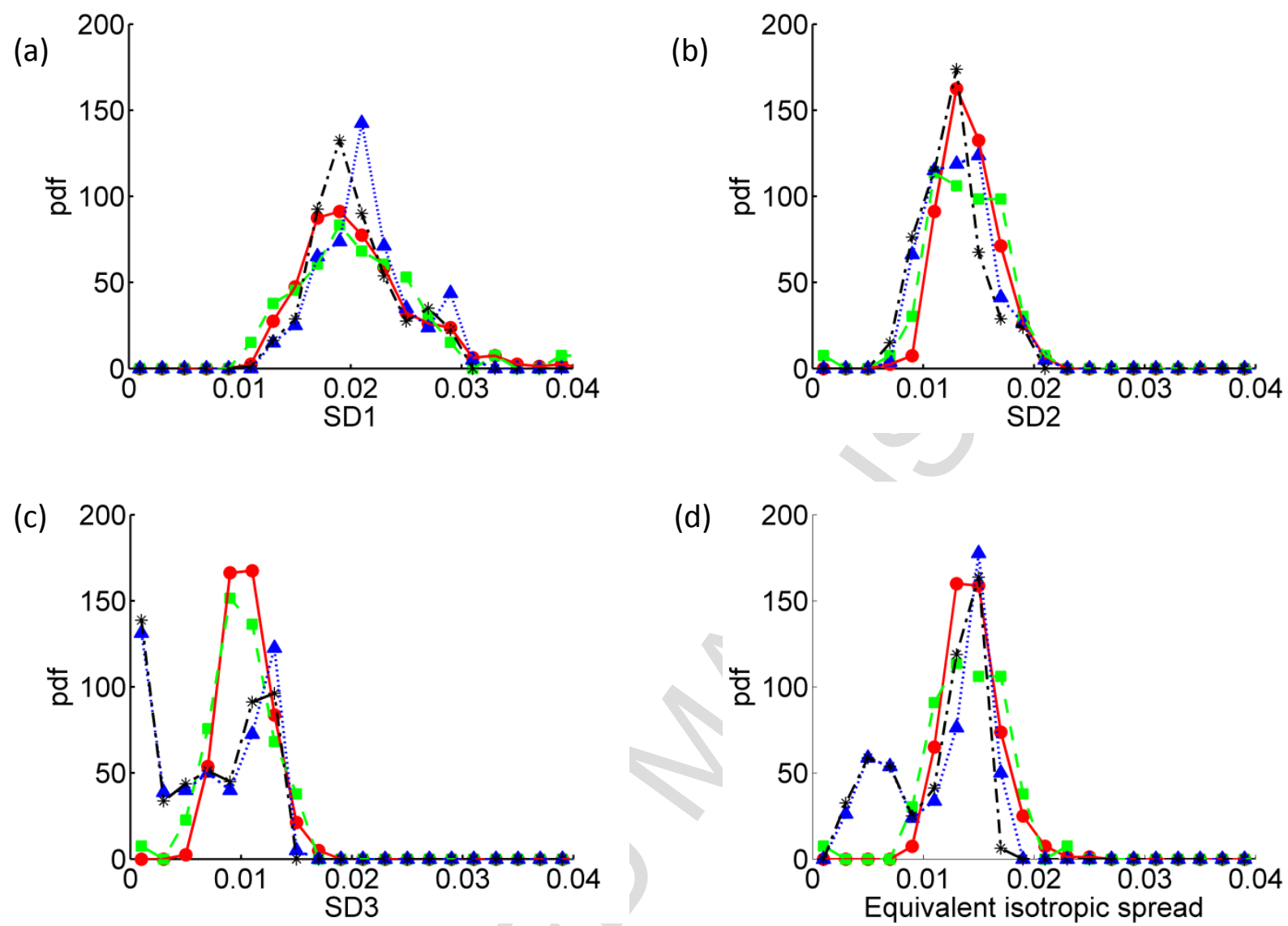

" Exp. - VPFFT \& Taylor expansion approx. * Discrete approx.

Figure 5. Probability density functions of the standard deviations along the principal axis of the misorientation distribution (a) SD1, (b) SD2, (c) SD3 and (d) equivalent isotropic spread for experimental results and simulation predictions for copper after $11 \%$ tensile strain. 


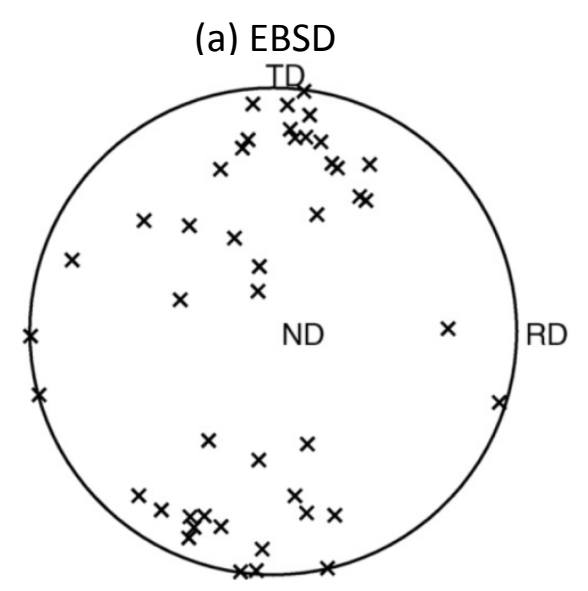

(c) Taylor expansion approx.

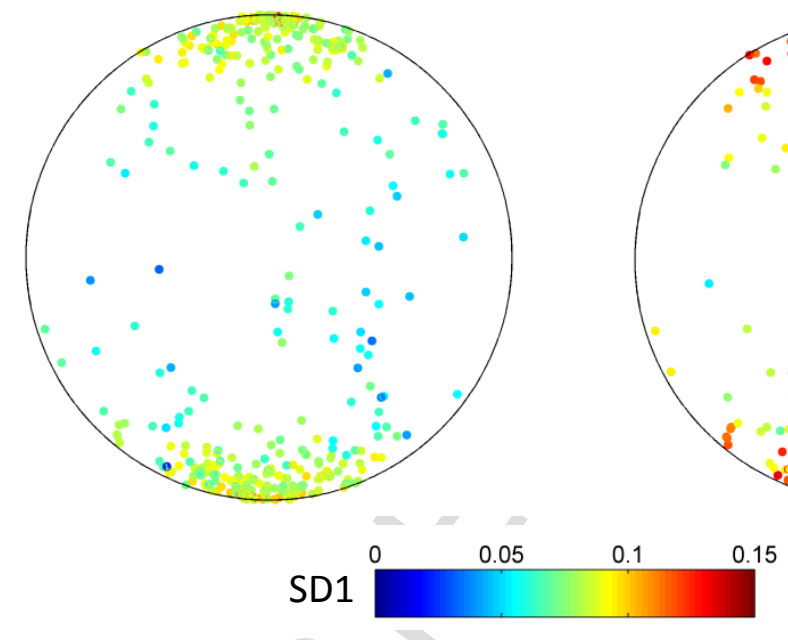

(b) VPFFT

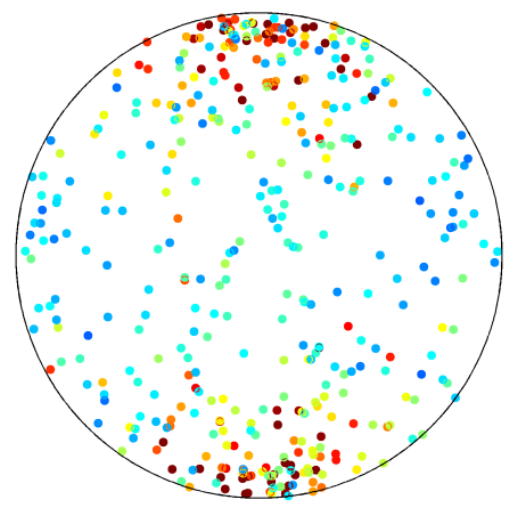

(d) Discrete approx.

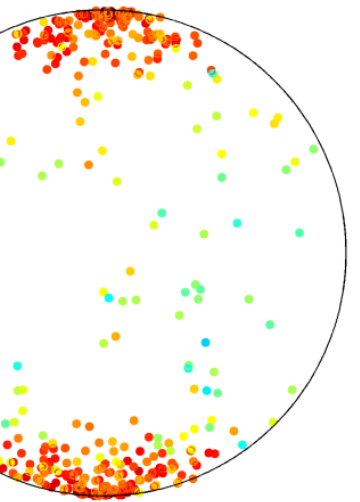

Figure 6. Dominant rotation axes plotted in the sample frame and color-coded according to magnitude of standard deviation along this direction for (a) EBSD data [44], (b) VPFFT results, (c) Taylor expansion approximation and (d) discrete approximation approach for aluminium after $38 \%$ plane strain compression or rolling reduction. 


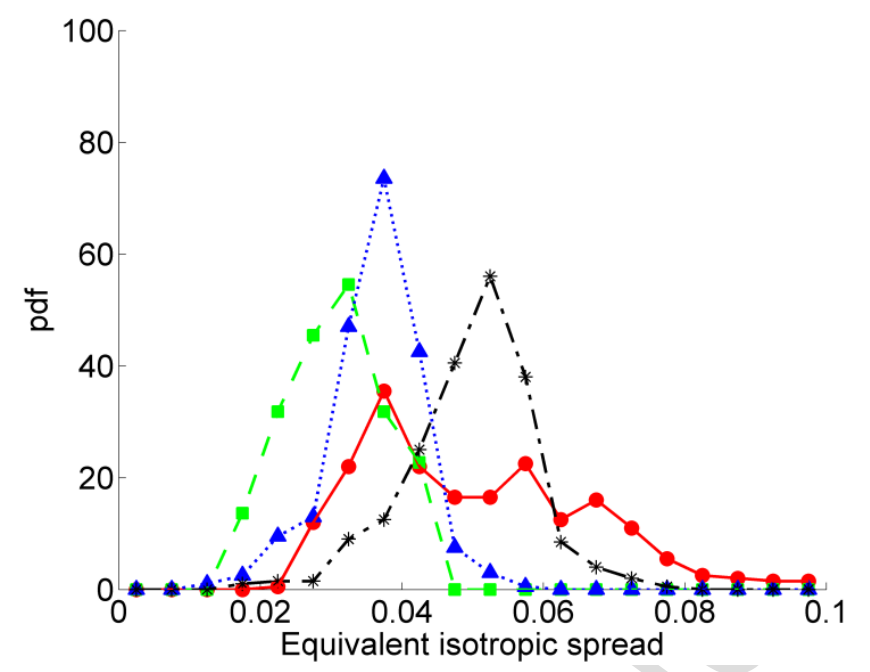

- Exp. • VPFFT ^ Taylor expansion approx. * Discrete approx.

Figure 7. Probability density functions of equivalent isotropic spread for experimental results and simulation predictions for aluminum after $38 \%$ plane strain compression or rolling reduction. 


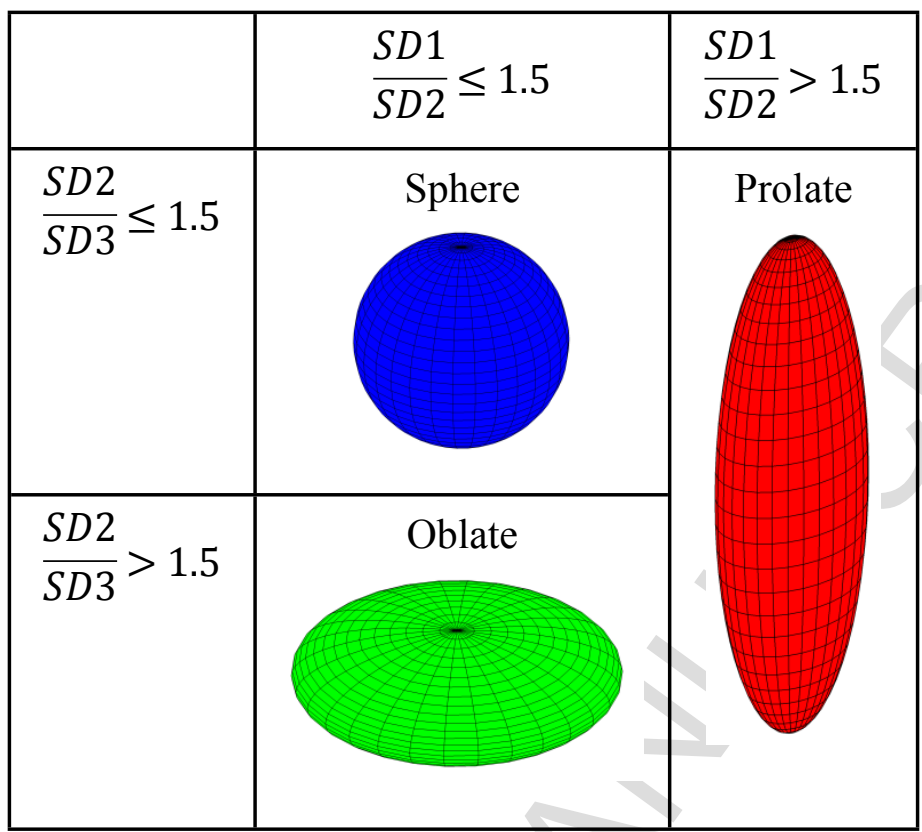

Table 1. Shapes of the misorientation distribution classified according to the ratios between standard deviations along principal directions. This classification is a variation of the one proposed by [41]. 


\begin{tabular}{|l|c|c|c|c|}
\hline & EBSD & VPFFT & $\begin{array}{l}\text { Taylor expan- } \\
\text { sion approx. }\end{array}$ & $\begin{array}{l}\text { Discrete } \\
\text { approx. }\end{array}$ \\
\hline Prolate & $42 \%$ & $40 \%$ & $50 \%$ & $50 \%$ \\
\hline Oblate & $23 \%$ & $17 \%$ & $24 \%$ & $24 \%$ \\
\hline Sphere & $35 \%$ & $43 \%$ & $26 \%$ & $26 \%$ \\
\hline
\end{tabular}

Table 2. Fractions of different distribution shapes after $11 \%$ tensile deformation of copper obtained from experimental data, VPFFT simulation, Taylor expansion approximation and discrete approximation approach. 


\begin{tabular}{|l|c|c|c|c|}
\hline $\begin{array}{l}\text { Fraction } \\
\text { of prolates }\end{array}$ & Exp. & VPFFT & $\begin{array}{c}\text { Taylor } \\
\text { expansion } \\
\text { approx. }\end{array}$ & $\begin{array}{c}\text { Discrete } \\
\text { approx. }\end{array}$ \\
\hline$[001] 10^{\circ}$ & $56 \%$ & $19 \%$ & $41 \%$ & $39 \%$ \\
\hline$[011] 20^{\circ}$ & $86 \%$ & $76 \%$ & $91 \%$ & $91 \%$ \\
\hline$[111] 15^{\circ}$ & $55 \%$ & $19 \%$ & $13 \%$ & $14 \%$ \\
\hline Remaining & $68 \%$ & $64 \%$ & $80 \%$ & $79 \%$ \\
\hline
\end{tabular}

Table 3. Fractions of prolate shapes for different texture components after $25 \%$ tensile deformation of copper obtained from experimental data [41], VPFFT simulation, Taylor expansion approximation and discrete approximation approach. 